\title{
Estrogens enhance myoblast differentiation in facioscapulohumeral muscular dystrophy by antagonizing DUX4 activity
}

\author{
Emanuela Teveroni, ${ }^{1,2}$ Marsha Pellegrino, ${ }^{1}$ Sabrina Sacconi, ${ }^{3,4}$ Patrizia Calandra, ${ }^{1}$ Isabella Cascino, ${ }^{1}$ Stefano Farioli-Vecchioli, ${ }^{1,5}$ \\ Angela Puma, ${ }^{3}$ Matteo Garibaldi, ${ }^{4,6}$ Roberta Morosetti, ${ }^{7}$ Giorgio Tasca, ${ }^{7}$ Enzo Ricci, ${ }^{7,8}$ Carlo Pietro Trevisan, ${ }^{9}$ Giuliana Galluzzi, ${ }^{1}$ \\ Alfredo Pontecorvi, ${ }^{2,10}$ Marco Crescenzi, ${ }^{11}$ Giancarlo Deidda, ${ }^{1}$ and Fabiola Moretti ${ }^{1,5}$

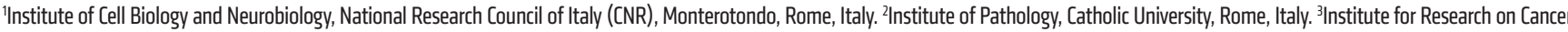
and Aging, INSERM, CNRS (IRCAN), Nice, France. ${ }^{4}$ Faculty of Medicine, University of la Côte d'Azur (UCA), Nice, France. ${ }^{5}$ IRCCS Fondazione Santa Lucia, Rome, Italy. ${ }^{6}$ Unit of Neuromuscular Diseases, Department of Neurology, Mental Health and Sensory Organs (NESMOS), "Sapienza” University of Rome, Rome, Italy. ${ }^{7}$ Neuroscience Area, Fondazione Policlinico Universitario "A. Gemelli," Rome, Italy. ${ }^{8}$ Institute of Neurology, Catholic University, Rome, Italy. ${ }^{9}$ Department of Neuroscience, University of Padua, Padua, Italy. ${ }^{10}$ Endocrinology and Metabolism Area, Fondazione Policlinico Universitario "A. Gemelli," Rome, Italy. "National Institute of Health, Rome, Italy.
\end{abstract}

Facioscapulohumeral muscular dystrophy (FSHD) is an autosomal dominant neuromuscular disorder that is characterized by extreme variability in symptoms, with females being less severely affected than males and presenting a higher proportion of asymptomatic carriers. The sex-related factors involved in the disease are not known. Here, we have utilized myoblasts isolated from FSHD patients (FSHD myoblasts) to investigate the effect of estrogens on muscle properties. Our results demonstrated that estrogens counteract the differentiation impairment of FSHD myoblasts without affecting cell proliferation or survival. Estrogen effects are mediated by estrogen receptor $\beta$ (ER $\beta$ ), which reduces chromatin occupancy and transcriptional activity of double homeobox 4 (DUX4), a protein whose aberrant expression has been implicated in FSHD pathogenesis. During myoblast differentiation, we observed that the levels and activity of DUX4 increased progressively and were associated with its enhanced recruitment in the nucleus. ER $\beta$ interfered with this recruitment by relocalizing DUX4 in the cytoplasm. This work identifies estrogens as a potential disease modifier that underlie sex-related differences in FSHD by protecting against myoblast differentiation impairments in this disease.

\section{Introduction}

Facioscapulohumeral muscular dystrophy (FSHD) is an autosomal dominant neuromuscular disorder with a prevalence of 1:20,000 to 1:15,000 worldwide (1). The major form of FSHD (FSHD1A, OMIM \#158900) is associated with a reduction of subtelomeric repeats (D4Z4) on the long arm of chromosome 4 (4q35). The normal polymorphic allele ranges from 11 to $100 \mathrm{D} 4 \mathrm{Z} 4$ units, whereas the pathologic one ranges from 1 to 10 units (1).

Clinical symptoms of FSHD range from mild (weakness of limited muscles) to severe (wheelchair dependence), with most of the affected individuals showing symptoms by age 20 (1). A subset of individuals (21\%) do not even show clinical signs (asymptomatic carriers) or are minimally affected. Extreme variability is present even in members of the same family carrying the same repeat array size, especially when the fragment size is in the higher range (2).The interfamilial variability of clinical symptoms is in part explained by the extent of D4Z4 contraction; indeed, a roughly inverse correlation exists between the number of residual D4Z4

Authorship note: G. Deidda and F. Moretti contributed equally to this work. Conflict of interest: The authors have declared that no conflict of interest exists. Submitted: July 6, 2016; Accepted: January 12, 2017.

Reference information: J Clin Invest. 2017;127(4):1531-1545.

https://doi.org/10.1172/JCl89401. units and the severity of clinical phenotype. Intrafamilial variability is still largely unexplained. Sex is one of the factors affecting sensitivity to the disease, with females being less severely affected than males and presenting a higher proportion of asymptomatic carriers, especially in the presence of a mild/long allele (3-5). The reduced correlation between the fragment size and age-corrected clinical severity score in female compared with male patients suggests the existence of specific disease modifiers in women (6). Additionally, no correlation has been found between sex and severity of phenotype in infantile FSHD patients (7). Although these patients usually carry short repeat arrays associated with high disease penetrance (8), a possible role for adult sex-related factors in the clinical presentation of the disease can be hypothesized. Furthermore, some studies have reported a persistent worsening of symptoms after childbirth in a subset of female FSHD patients (9-11). Since a strong drop of hormone levels characterizes this condition, a plausible hypothesis is that female hormones and possibly estrogens may be involved in FSHD disease (12).

At the cellular level, FSHD myoblasts are characterized by (a) impaired differentiation with reduced fusion index and altered myotube structure; (b) increased sensitivity to oxidative stress; and (c) reduced viability of differentiated myotubes due to increased cell death and/or increased atrophy (13). The molecular mechanisms that underlie these phenomena are incomplete- 

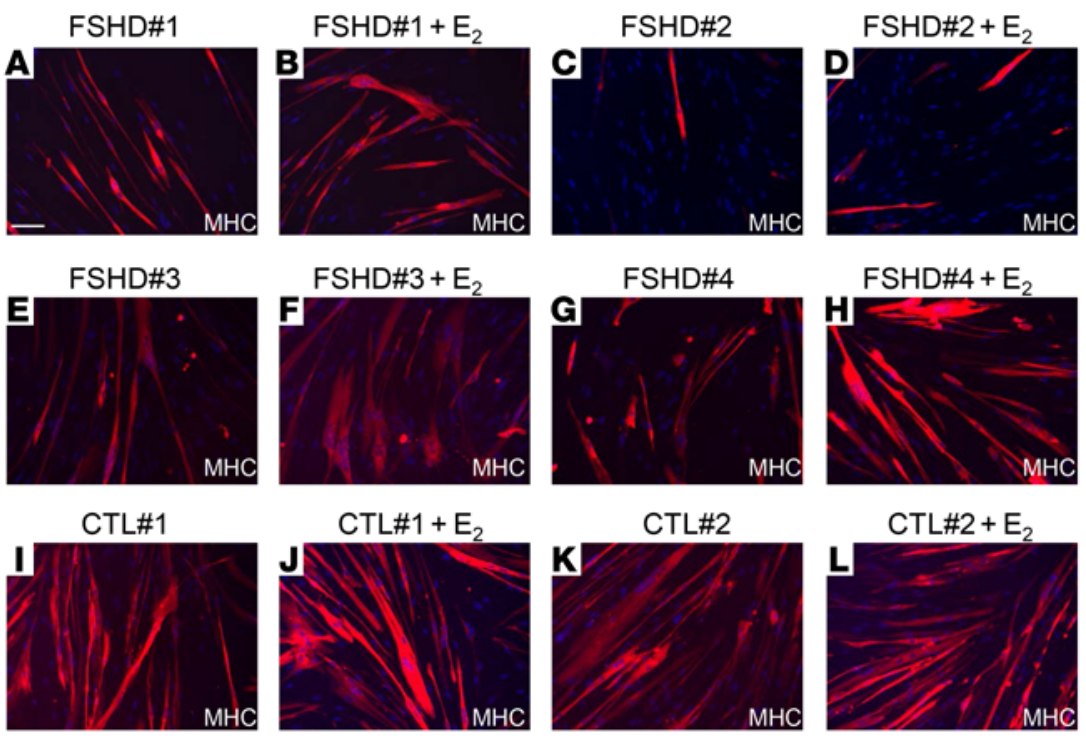
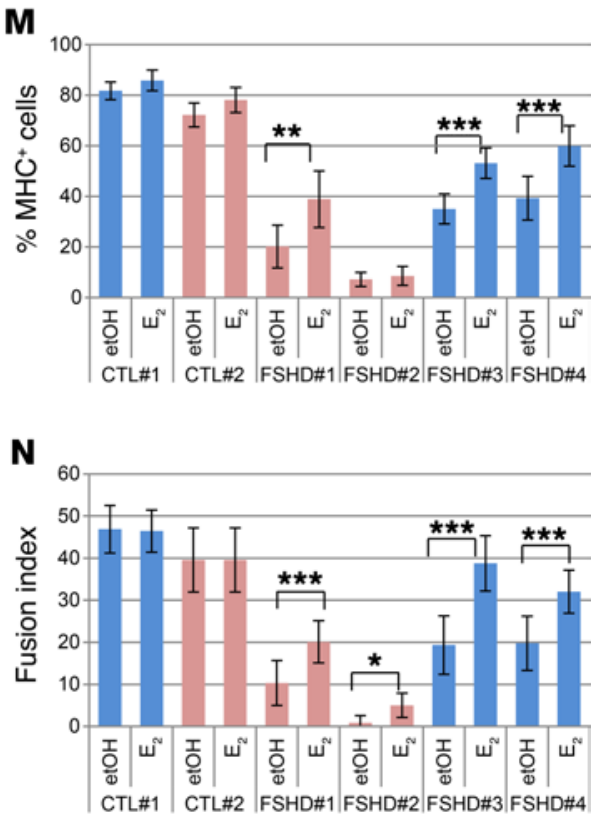

Figure 1. $E_{2}$ enhances differentiation of immortalized FSHD myoblasts. (A-L) Representative photographs of MHC immunostaining (red) of myoblasts from indicated FSHD patients and healthy individuals after 7 days of culture in differentiation medium in the absence or presence of $10^{-8} \mathrm{M} \mathrm{E}_{2}$. Nuclei were counterstained with DAPI (blue). Scale bar: $75 \mu \mathrm{m}$. (M) Percentage of $\mathrm{MHC}^{+}$cells treated as in $\mathbf{A}-\mathbf{L}$. Percentage $\mathrm{MHC}^{+}$cells $=\mathrm{MHC}^{+}$nuclei/total number of nuclei. Pink bars represent female, blue bars male myoblasts. (N) Fusion index in the same fields analyzed in $\mathbf{M}$. Fusion index = percentage of nuclei in $\mathrm{MHC}+$ myotube/number of total nuclei, where a myotube is a $\mathrm{MHC}^{+}$cell with 3 nuclei. Data in $\mathbf{M}$ and $\mathbf{N}$ represent the mean $\pm \mathrm{SD}$ of 3 independent experiments, except 2 experiments are represented for FSHD\#2 and CTLs. For each experiment, 4 fields/condition were counted ( $n=12$ for FSHD\#1, FSHD\#3, and FSHD\#4; $n=8$ for FSHD\#2, CTL\#1, and CTL\#2). ${ }^{* * *} P<0.001 ;{ }^{* *} P<0.01 ;{ }^{*} P<0.05,2$-tailed Student's $t$ test. The complete set of fields evaluated in this experiment is reported in Supplemental Figure 6.

ly defined. The current molecular pathogenetic model of FSHD1 proposes that contraction of the D4Z4 array is accompanied by DNA hypomethylation (14-16) that leads to a decreased heterochromatinization of the region. In turn, this leads to the transcription of usually repressed genes. Among these, double homeobox 4 (DUX4), mainly expressed by the last D4Z4 unit, is the best FSHD candidate gene. DUX4 is a transcription factor that regulates genes involved in stem cell and germline development (17). Its aberrant expression in myoblasts alters factors involved in muscle differentiation, oxidative stress homeostasis, and muscle atrophy in addition to immune response and RNA surveillance, therefore recapitulating the key features of FSHD myopathy (18). At present, there are no studies, to our knowledge, reporting estrogen activity toward DUX4 levels and/or function.

Estrogen activity is mediated mainly by 2 nuclear receptors: estrogen receptor $\alpha(E R \alpha)$ and estrogen receptor $\beta(E R \beta)$. The characterization of the 2 receptors in the human muscle has provided conflicting results. Indeed, the mRNAs of the ER $\alpha$ and ER $\beta$ genes ( $E S R 1$ and $E S R 2$, respectively) have been reported in human muscle $(19,20)$. However, the presence of $E R \alpha$ protein has not been definitely proved $(20,21)$, raising the possibility that ESR1 is not expressed in human muscle cells and that the mRNA signal derives from environmental cells (i.e., endothelial cells, fibroblasts). Conversely, the expression of ER $\beta$ in human myoblasts has been shown by immunohistochemistry (IHC) (22).

In this work, we investigated the effect of estrogens in FSHD and demonstrated that estrogens improve the differentiation properties of FSHD-derived myoblasts by antagonizing DUX4 activity.

\section{Results}

Estrogens enhance in vitro differentiation of FSHD-derived myoblasts. To study the activity of estrogens in FSHD, we used myoblasts isolated from affected patients compared with myoblasts from healthy individuals. Four myoblast cell cultures were obtained from patients affected by FSHD1, 2 females (hereafter FSHD\#1 and FSHD\#2) and 2 males (FSHD\#3 and FSHD\#4). Control myoblast cell cultures were derived from 1 healthy male (CTL\#1) and 2 females (CTL\#2 and CTL\#3). Features related to these individuals are reported in Supplemental Table 1 (supplemental material available online with this article; doi:10.1172/JCI89401DS1). Given the short in vitro life span of FSHD primary cells, control and FSHD myoblasts were immortalized by retroviral infection with the CDK4-R24C variant and hTERT expression vectors (23). Analysis of cell positivity to the myoblast lineage marker desmin revealed that all immortalized cell cultures maintained $90 \%$ or more cells positive for desmin, similar to primary cells (Supplemental Figure 1, A and B), indicating that the immortalization procedure did not alter myoblast phenotype. As a control, primary human fibroblasts were entirely negative to this marker (Supplemental Figure 1B). Moreover, the immortalization did not prevent the differentiation of myoblasts, as indicated by the positivity to the marker of terminal differentiation, myosin heavy chain (MHC) (Supplemental Figure 1C).

Isolated FSHD myoblasts are characterized by altered differentiation and fusion in vitro (24-27). To ascertain whether estrogens play a protective role in FSHD, we analyzed their ability to improve FSHD muscle differentiation. To this aim, immortalized myoblasts 
A

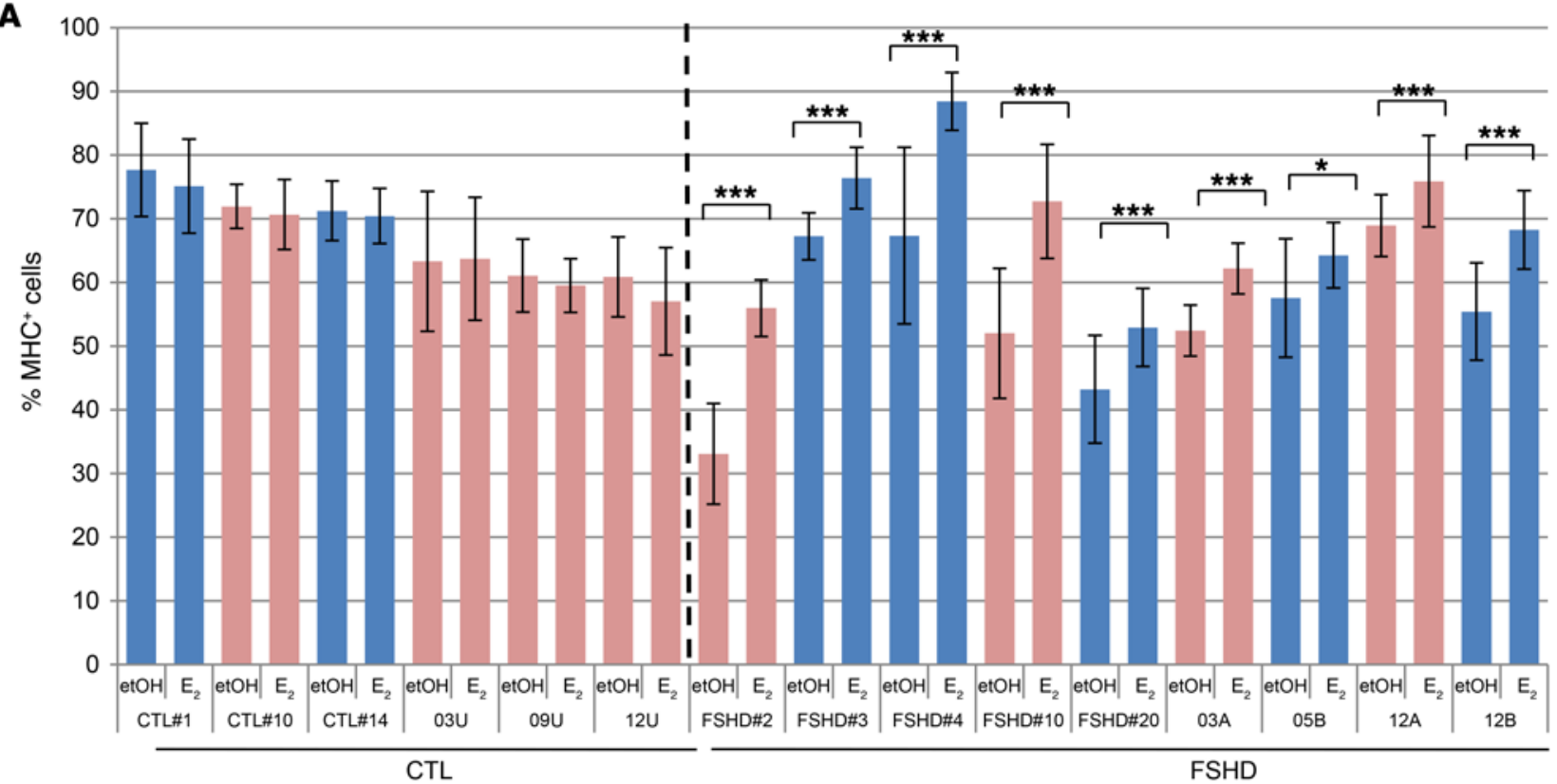

B

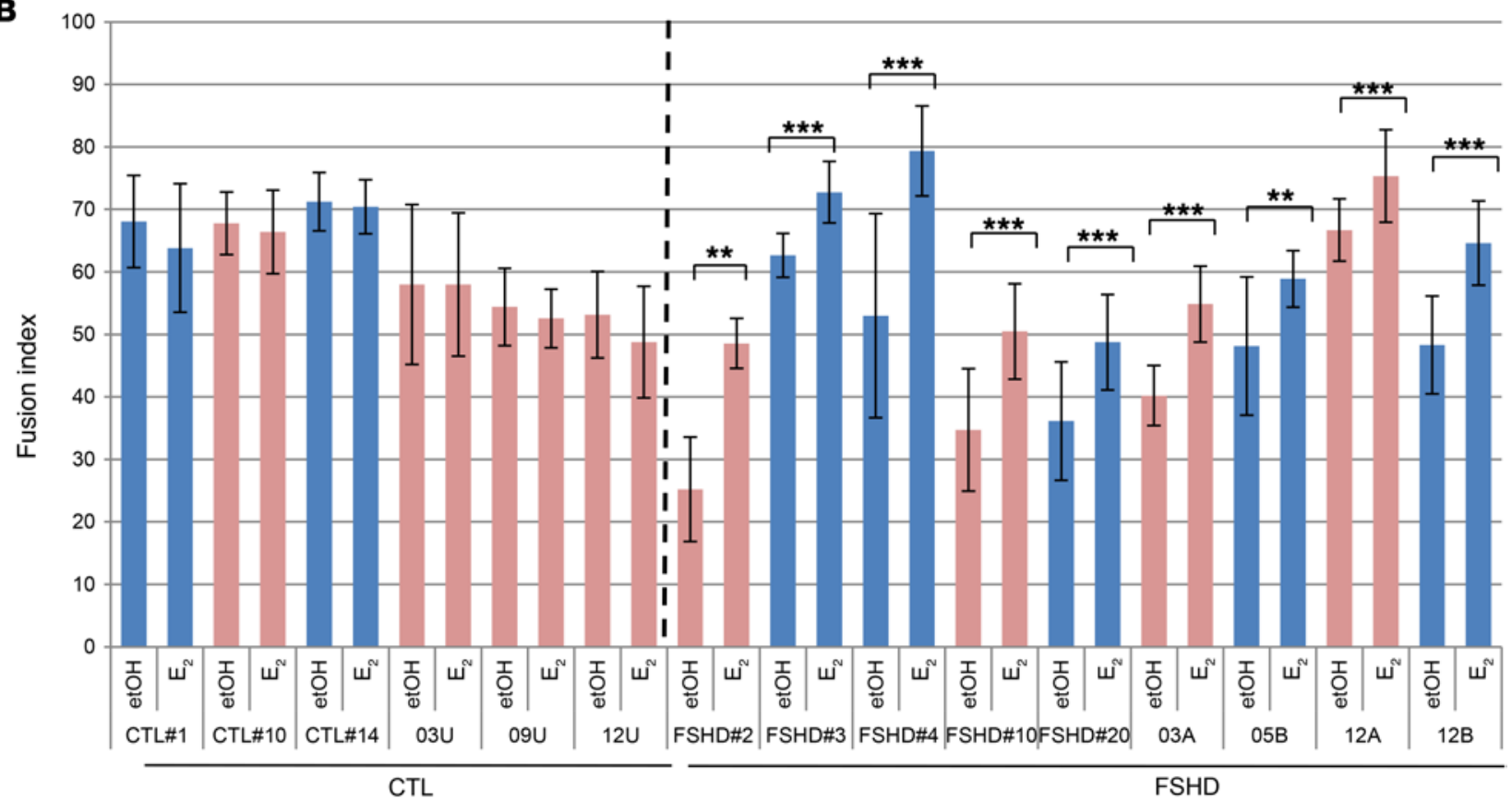

Figure 2. $\mathrm{E}_{2}$ enhances differentiation of primary FSHD-isolated myoblasts. (A) Percentage of $\mathrm{MHC}^{+}$myoblasts from indicated healthy individuals and FSHD patients after 7 days of culture in differentiation medium in the absence or presence of $10^{-8} \mathrm{ME}_{2}$. Percentage of $\mathrm{MHC}^{+}$cells $=\mathrm{MHC}^{+}$nuclei/total number of nuclei. Pink bars represent female, blue bars male myoblasts. (B) Fusion index in the same fields evaluated in $\mathbf{A}$. Fusion index $=$ percentage of nuclei in $\mathrm{MHC}+$ myotube/number of total nuclei, where a myotube is an $\mathrm{MHC}^{+}$cell with 3 or more nuclei. Data in $\mathbf{A}$ and $\mathbf{B}$ represent the mean \pm SD of 2 independent experiments, but 1 experiment is represented for CTL\#1, FSHD\#2, and FSHD\#3. For each experiment, 8 fields/condition were counted. ( $n=8$ for CTL\#1, FSHD\#2, and FSHD\#3; $n=16$ for all other myoblast cell lines). ${ }^{* * *} P<0.001 ;{ }^{*} P<0.01 ;{ }^{*} P<0.05,2$-tailed Student's $t$ test. A subset of 8 fields/condition for all FSHD and CTL\#14 and 03U myoblasts evaluated in this experiment is reported in Supplemental Figure 6.

were differentiated in the presence or absence of $17 \beta$-estradiol $\left(\mathrm{E}_{2}\right)$, the predominant hormone in terms of estrogenic activity. Cells were plated at high confluence $\left(>90 \%, 300\right.$ cells $\left./ \mathrm{mm}^{2}\right)$ and grown for 16 hours in medium deprived of estrogens and estrogen-like substances. Afterward, cultures were switched to differentiation medium deprived of estrogen and estrogen-like substances (hereafter Diff) in the presence or absence of $\mathrm{E}_{2}$. The medium was replaced every other day, and after 1 week, cells were immunostained for MHC (Figure 1, A-L). The overall differentiation of the immortalized FSHD myoblasts was decreased compared with that of healthy controls (compare Figure 1, A-H to I-L, and Figure 1, M and $\mathrm{N}$ ). Interestingly, $\mathrm{E}_{2}$ increased the number of differentiated cells in comparison with cells treated with vehicle (etOH) independently of the sex of patients providing FSHD myoblasts and the 
fragment length (Figure 1M and Supplemental Table 1). Particularly, a significant increase in the number of multinucleated myotubes was evident in all FSHD cell cultures (Figure 1N). Importantly, in control myoblasts, no significant change was evident, indicating that the observed effects are specific for FSHD myoblasts (Figure 1, $\mathrm{I}-\mathrm{N})$. The average density of nuclei $/ \mathrm{mm}^{2}$ in the FSHD samples (243 \pm 48 ) was reduced compared with that of controls (316 \pm 8$)$, but was not significantly different between etOH and $\mathrm{E}_{2}$ (FSHD, $228 \pm 36$ vs. $258 \pm 60$; CTL, $319 \pm 10$ vs. $314 \pm 8$ ).

To ascertain that these effects are not related to the immortalization procedure and to confirm these data in additional cell lines, previous differentiation conditions were applied to primary FSHD and control myoblasts isolated from 2 different centers. Compared with the previous immortalized cell lines, primary FSHD myoblasts showed a distribution of differentiation and fusion index closer to that of healthy individuals (Figure 2, A and B). Particularly, primary FSHD\#2, FSHD\#3, and FSHD\#4 myoblasts showed increased differentiation properties compared with their immortalized counterparts (see Figure 1, M and N), suggesting that the immortalization procedure reduces FSHD differentiation potential. Importantly, $\mathrm{E}_{2}$ treatment significantly improved differentiation capabilities of all primary FSHD myoblasts while not affecting those of controls (Figure 2, A and B), thus confirming previous results and highlighting a particular sensitivity of FSHD myoblasts to estrogens. No significant difference was observed in the average density of nuclei/ $/ \mathrm{mm}^{2}$ between etOH and $\mathrm{E}_{2}$ treatment of each control or FSHD cell line (Supplemental Figure 2A). To conservatively analyze these results and avoid the influence of the FSHD-reduced differentiation in the evaluation of the fusion index, we also analyzed the percentage of nuclei in myotubes related to the number of $\mathrm{MHC}^{+}$nuclei (corrected fusion index $\left[\mathrm{FI}_{\text {corr }}\right]$ ) rather than to the total number. This parameter was still significantly enhanced by estrogens in FSHD myoblasts while not in control cells (Supplemental Figure 2B).

One of the well-established activities of estrogens is their proproliferative/prosurvival action, mainly attributed to $\mathrm{ER} \alpha(28$, 29). We therefore wondered whether the $E_{2}$-enhanced differentiation was related to an increased number of differentiating cells. Previous data do not indicate a significant difference in the final number of etOH- and $\mathrm{E}_{2}$-treated differentiated cells. Further time-course analysis of cell growth in different primary and immortalized cell lines showed that $\mathrm{E}_{2}$ treatment did not increase the number of FSHD and control myoblasts during differentiation (Supplemental Figure 2, C-E) or in proliferative conditions (Supplemental Figure 2F). These data exclude that the $\mathrm{E}_{2}$-mediated enhanced differentiation of FSHD myoblasts is due to increased proliferation, thus supporting a specific activity of these hormones toward FSHD myoblasts.

Estrogens antagonize DUX4-mediated impairment of myoblast differentiation. The expression of full-length DUX4 is considered the main pathogenic factor of muscular dystrophy in FSHD patients $(25,30)$. We, therefore, tested whether estrogens specifically counteract DUX4-mediated activity. Control myoblasts were transfected with DUX4 coding sequence in frame with the V5 tag at the C-terminal (hereafter DUX4-V5) (31), and after 5 days of growth in the Diff medium with or without $\mathrm{E}_{2}$, myotube formation was verified by analysis of MHC immunofluores- cence. In the absence of $\mathrm{E}_{2}, \mathrm{DUX} 4-\mathrm{V} 5$ overexpression led to a strong decrease in the number of MHC-positive cells, supporting its activity in impairing muscle differentiation (Figure 3, A and $B$, and refs. 24, 32). Of note, the presence of $E_{2}$ antagonized this effect and was able to efficiently recover the differentiation impairment caused by DUX4 whereas it did not affect the differentiation of cells transfected with control vector (Figure 3, A and B). Western blot of myotubes after 1 and 3 days of differentiation confirmed the continued expression of DUX4-V5, substantially not altered by $\mathrm{E}_{2}$ (Figure $3 \mathrm{C}$ ), suggesting that estrogens do not function by altering the protein levels of DUX4. Since it has been reported that DUX4 is degraded through the proteasome system (25), to confirm further that estrogens do not affect DUX4 protein stability, we analyzed DUX4 in the presence or absence of proteasome inhibitor MG132. Under the conditions used in our experiments, MG132 did not stabilize and rather decreased the levels of DUX4-V5. Importantly, $\mathrm{E}_{2}$ did not further alter these levels (Figure 3D), confirming that $\mathrm{E}_{2}$ does not modify DUX4-V5 levels. As a control, the levels of MDM2 and p21, 2 well-known targets of proteasome activity, were highly increased by MG132. To get more insights into the activity of $\mathrm{E}_{2}$ toward DUX4 and exclude any interference of the epitope tag, the myoblast differentiation experiments were repeated by expressing DUX4 in frame with the mCherry protein at the $\mathrm{N}$ terminus (hereafter Cherry-DUX4). In the absence of $\mathrm{E}_{2}$, the double fluorescence of the mCherry and the MHC signals confirmed the substantial reduction of MHC-positive cells in DUX4-expressing cells (Figure $3 \mathrm{E}$ ), confirming the impairment of myoblast differentiation by DUX4. Once again, $\mathrm{E}_{2}$ significantly improved the differentiation properties of myoblasts (Figure 3, E and F). Overall, these data indicate the ability of estrogens to antagonize DUX4-mediated impaired muscle differentiation.

Overexpression of DUX4 has been associated with cell death in murine and human muscle cells $(31,33,34)$. To exclude that $\mathrm{E}_{2}$ is acting as a prosurvival factor, the Cherry-DUX4 transfected cells were analyzed for the expression of the apoptosis marker annexin V. The number of the double-annexin V/mCherry-positive cells was indeed increased in Cherry-DUX4 compared with mCherry-transfected cells, independently of cell growth conditions, confirming the toxicity of overexpressed DUX4. However, this fraction was not significantly altered by $\mathrm{E}_{2}$ treatment (Supplemental Figure 3), thus excluding a prosurvival effect of $\mathrm{E}_{2}$ and confirming its ability to counteract specifically the differentiation impairment caused by DUX4.

$E R \beta$ mediates estrogen-enhanced differentiation. $\mathrm{E}_{2}$ is the natural ligand of ER $\alpha$ and ER $\beta$. To understand which receptor underlies the $\mathrm{E}_{2}$ activity in FSHD myoblasts, $\mathrm{ER} \alpha$ and $\mathrm{ER} \beta$ expression were analyzed in control and FSHD myoblasts. Quantification of mRNA for ESR1 and ESR2 genes showed that the levels of ESR1 were very low in all myoblast populations as compared with the levels of ER $\alpha$-positive breast cell line MCF7, set to 1 (fold of reduction $\geq 80$ ) and were comparable to the levels of ER $\alpha$-negative MDA-MB-231 cells (ref. 35 and Figure 4A). Accordingly, no detectable levels of $\mathrm{ER} \alpha$ protein by Western blot were observed in primary cells (Supplemental Figure 4A), in agreement with previous data (20). Since ER $\alpha$ mainly mediates proliferation, these data well conform with the lack of $\mathrm{E}_{2}$ proliferative activity in these cells. Conversely, the 
A $E_{2}$ :
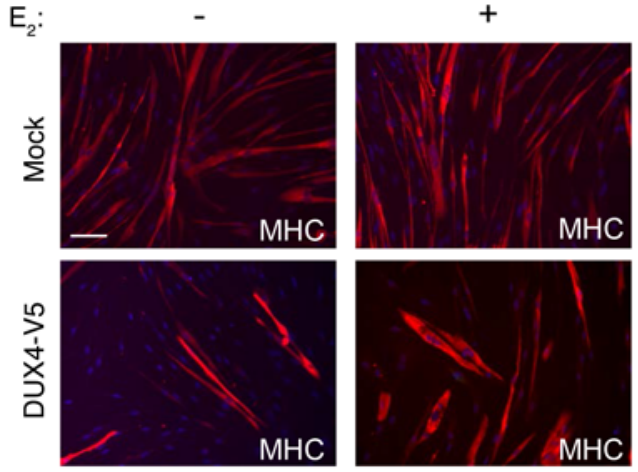

C

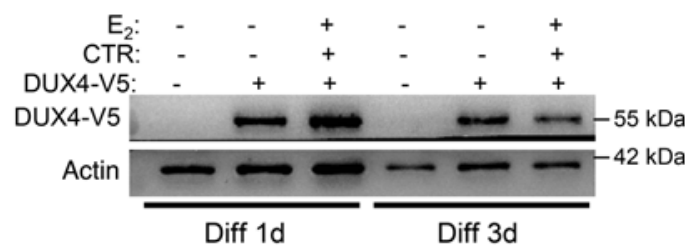

E $E_{2}$ :

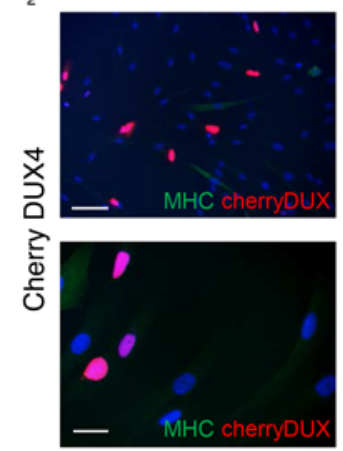

$+$
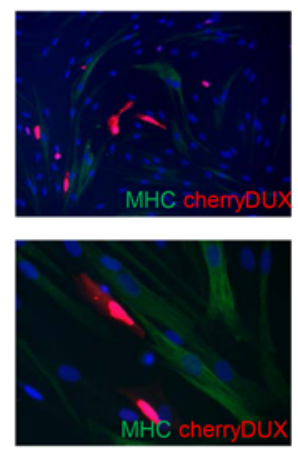

$20 X$

B

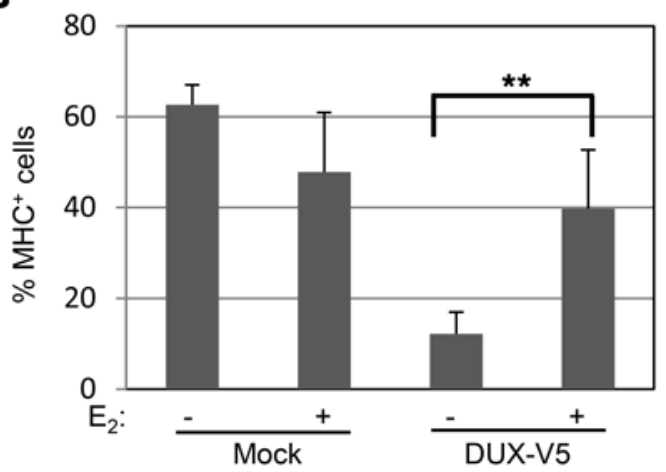

D

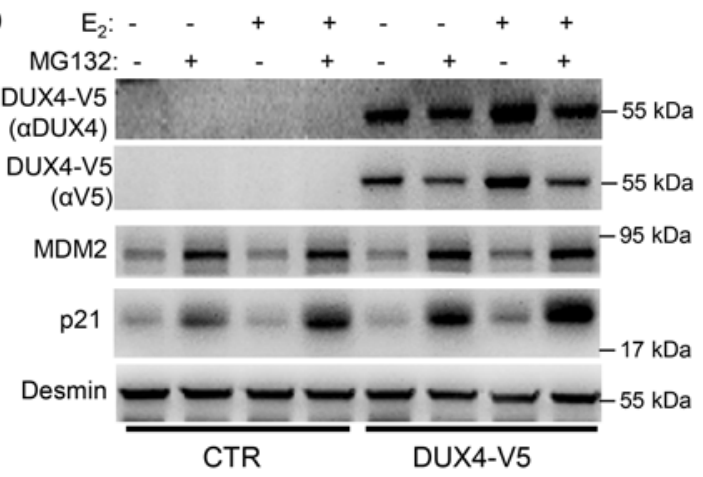

$\mathbf{F}$

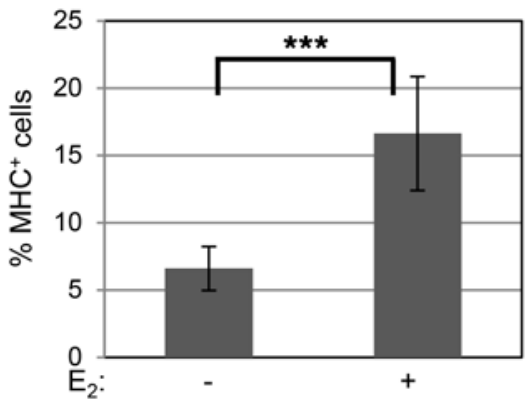

Figure 3. $\mathrm{E}_{2}$ antagonizes DUX4-mediated impairment of myoblast differentiation. (A) Representative photographs of MHC immunostaining (red) in control myoblasts transfected with empty vector (mock, upper panels) or DUX4-V5 (lower panels) after 4 days of culture in differentiation medium in the absence or presence of $E_{2}$. Nuclei were counterstained with DAPI (blue). Scale bar: $75 \mu \mathrm{m}$. (B) Percentage of $\mathrm{MHC}^{+}$cells treated as in $\mathbf{A}$. Mean \pm SD of 2 independent experiments is shown. Three different fields for each condition were counted $(n=6)$. (C) Western blot of the indicated proteins in the lysates from myoblasts treated as in A. DUX4-V5 was detected by aV5 antibody. (D) Western blot of the indicated proteins in control myoblasts transfected as in A and collected after 72 hours of culture in differentiation medium with or without $E_{2}$ and with $60 \mu \mathrm{M} \mathrm{MG132}$ for the last 4 hours. See complete unedited blots for $\mathbf{C}$ and $\mathbf{D}$ in the supplemental material. (E) Representative photographs of MHC immunostaining (green) in control myoblasts transfected with Cherry-DUX4 (red) after 3 days of culture in differentiation medium in the absence or presence of $E_{2}$. Nuclei were counterstained with DAPI (blue). Scale bars: $75 \mu \mathrm{m}$ (upper panels); $25 \mu \mathrm{m}$ (lower panels). (F) Quantification of double MHC ${ }^{+}$Cherry-Dux cells. Mean \pm SD of 2 independent experiments is shown. Four different fields for each condition were counted $(n=8) .{ }^{* *} P<0.01 ;{ }^{* *} P<0.001,2$-tailed Student's $t$ test.

levels of ESR2 were comparable to those of the ER $\beta$-positive ovarian cancer cell line A2780 (36), independently of the sex (Figure $4 \mathrm{~B})$, suggesting that ER $\beta$ is expressed in all human myoblasts. In fact, Western blot confirmed the presence of the protein in primary FSHD and control myoblasts (Supplemental Figure 4A). To ascertain that ER $\beta$ is transcriptionally active in these cells, control myoblasts were transfected with a luciferase vector whose expression was driven by estrogen responsive elements (ERE). Treatment with $E_{2}$ led to a sharp increase in luciferase activity, which was counteracted by the addition of tamoxifen, an antagonist of estrogen receptor activity (Figure $4 \mathrm{C}$ ). The overexpression of
FLAG-tagged ER $\beta$ (ER $\beta F L A G$ ) as positive control confirmed these data (Figure 4, C and D). Of note, $\mathrm{E}_{2}$ decreased the protein levels of ER $\beta$ both endogenously and exogenously expressed, whereas tamoxifen antagonized this reduction (Figure 4D). This decrease occurred as a negative feedback when estrogen receptors were stimulated by their ligand in estrogen-responsive tissues, whereas this phenomenon does not occur in the absence of estrogen-mediated transcriptional activity (37). These data, therefore, further support the presence of active ER $\beta$ in these cells. Overall, these data demonstrate that ER $\beta$ is $\mathrm{E}_{2}$ sensitive and functioning in these cells and suggest its involvement in $\mathrm{E}_{2}$ activity on FSHD myoblast 
A

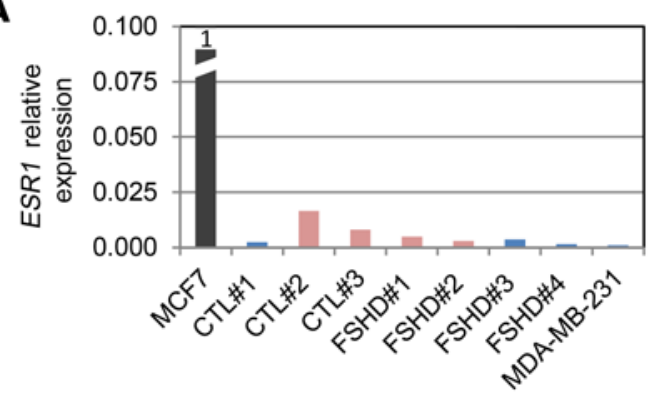

C

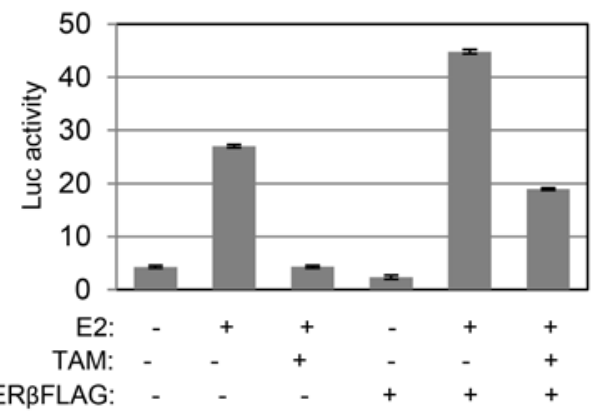

B

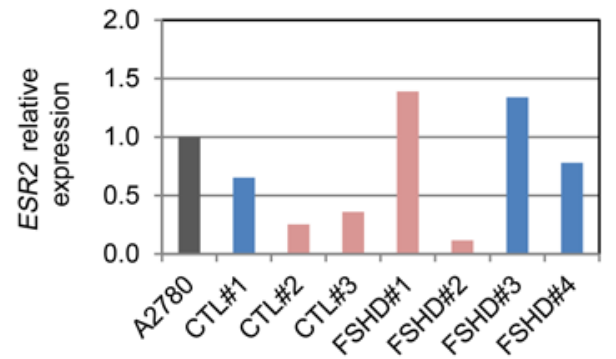

D

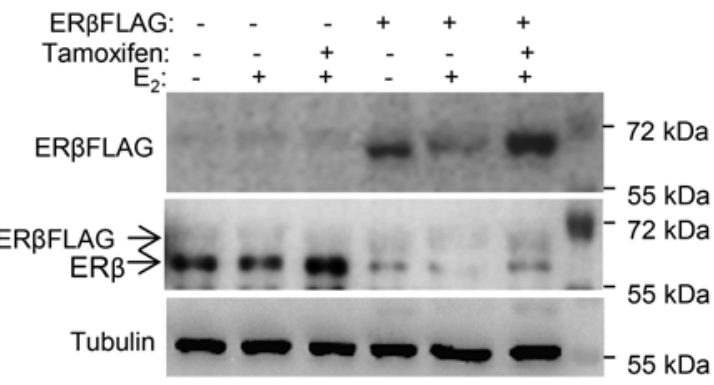

E

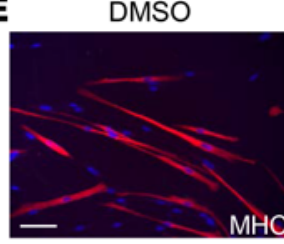

3ß-diol

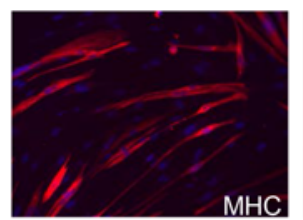

DPN

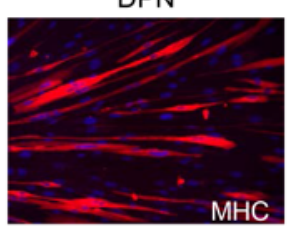

$\mathrm{E}_{2}$

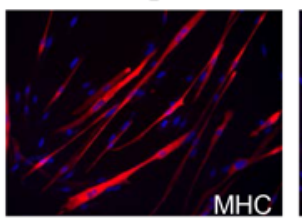

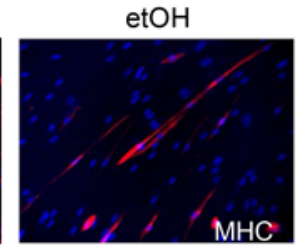

$E_{2}+$ TAM

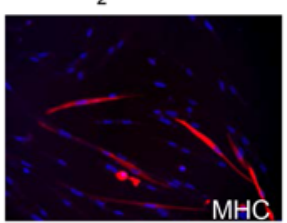

F

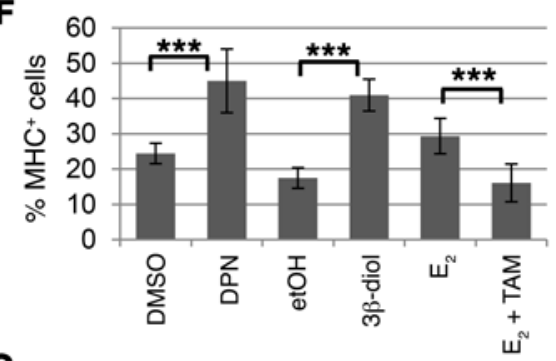

G

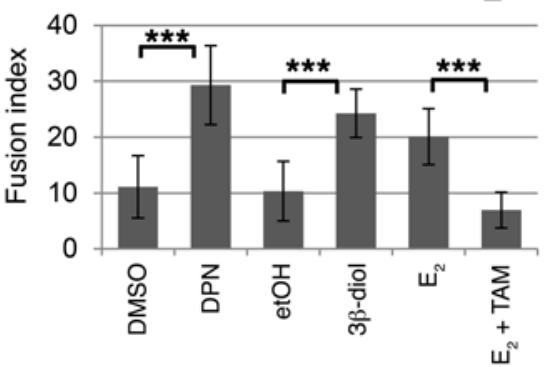

Figure 4. ERI3 mediates estrogen-enhanced differentiation. (A and B) mRNA levels by qRT-PCR of ESR1 (encoding ER $\alpha$ ) (A) and ESR2 (encoding ER $\beta$ ) (B) in immortalized cell lines. ESR1 levels in MCF7 (A) and ESR2 levels in A2780 (B) were set to 1 and the levels in myoblast cell lines relatively calculated. (C) Relative luciferase activity in CTL\#1 myoblasts transfected with estrogen-responsive element-luciferase vector (ERE-LuC) and/or ER $\beta F L A G$ as indicated, treated with or without $\mathrm{E}_{2} \pm$ tamoxifen. Luminescence signal was normalized to total protein content. Mean \pm SD of 2 independent experiments performed in triplicate is shown $(n=6)$. (D) Western blot analysis of endogenous ER and exogenous ER $\beta F L A G$ (whose position relative to ER is indicated by the arrows) in CTL\#1 myoblasts treated as indicated. See complete unedited blots in the supplemental material. (E) Representative photographs of MHC immunostaining (red) of myoblasts from FSHD\#1 patient after 7 days of culture in differentiation medium in the absence or presence of indicated treatments. Scale bar: $75 \mu \mathrm{m}$. (F and $\mathbf{G})$ Percentages of $\mathrm{MHC}^{+}(\mathbf{F})$ or fusion index $(\mathbf{G})$ of cells treated as in $\mathbf{E}$. Mean \pm SD of 3 independent experiments. Four different fields for each condition were counted $(n=12)$. ${ }^{* *} P<0.001,2$-tailed Student's $t$ test.

differentiation. To verify this hypothesis, 2 specific ER $\beta$ agonists were used: diarylpropionitrile (DPN), which has a 70-fold selectivity over $\operatorname{ER} \alpha$ (38), and a natural endogenous ligand of $\operatorname{ER} \beta, 5 \alpha$-androstane-3 $\beta, 17 \beta$-diol ( $3 \beta$-diol). Of note, both ligands were able to recapitulate and even increase the positive effect of $\mathrm{E}_{2}$ on differentiation of immortalized FSHD\#1 myoblasts (Figure 4E), both as a percentage of MHC-positive cells (Figure 4F) and as a fusion index (Figure $4 \mathrm{G}$ ), whereas cotreatment with tamoxifen antagonized the $\mathrm{E}_{2}$ beneficial effect (Figure $4, \mathrm{E}-\mathrm{G}$ ). Analysis of average nuclei numbers in each treatment by Kruskal-Wallis with Bonferroni's correction did not show any significant difference, thus excluding an evident proliferative activity of these hormones. Further time course analysis of cell proliferation of cells treated with the most effective ER $\beta$ agonist, DPN, did not evidence any alteration of this parameter (Supplemental Figure 4B). These data establish the ER $\beta$-mediated beneficial activity of estrogens on FSHD myoblast differentiation. Retrospective analysis of a limited number of female patients who underwent a rapid decline in estrogen lev- 
A

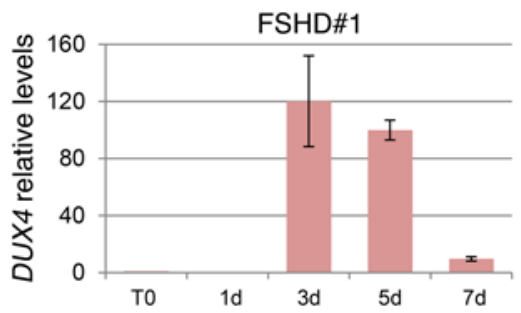

C

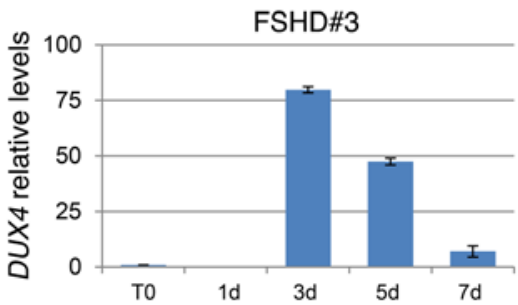

E

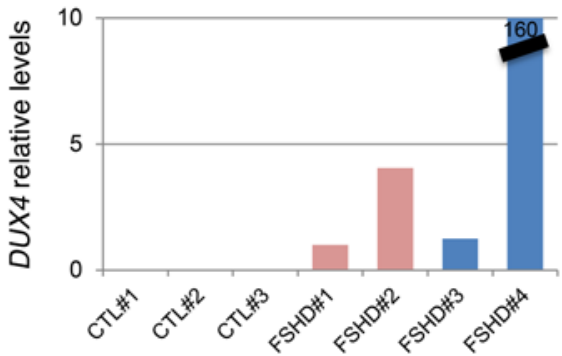

B

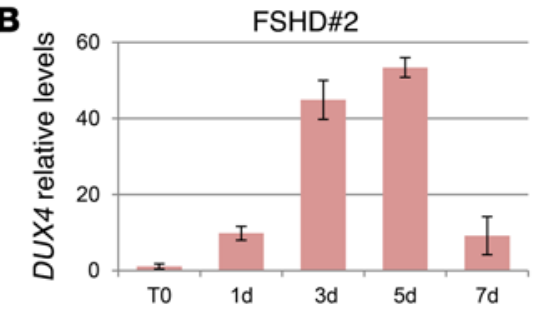

D

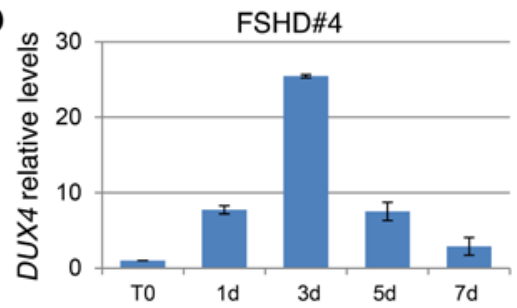

$\mathbf{F}$

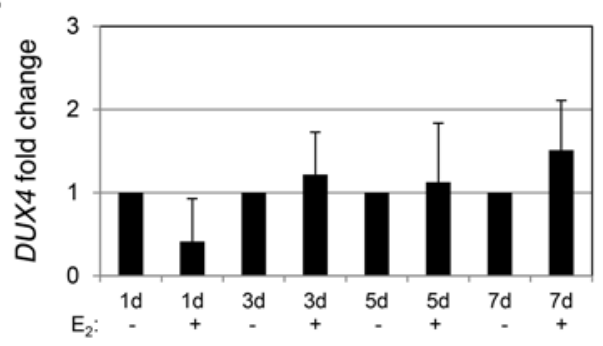

Figure 5. DUX4 levels increase during differentiation and are not affected by estrogens. (A-D) DUX4 mRNA levels by qRT-PCR in immortalized myoblasts derived from indicated patients, cultured in differentiation medium without estrogens at the indicated time points. The mRNA levels at the starting point of differentiation were set to 1 . Mean \pm SD of 2 independent experiments is shown. All samples were normalized to $G A P D H$. (E) DUX4 mRNA levels by qRT-PCR in proliferating immortalized myoblasts derived from indicated FSHD patients and healthy individuals. The mRNA levels of FSHD\#1 were arbitrarily set to 1. Data derive from 2 independent experiments. (F) Fold change of $D U X 4$ mRNA levels upon $E_{2}$ treatment at the indicated time points of differentiation. The mRNA levels upon etOH treatment at each time point were set to 1 . Mean \pm SD of data from 4 FSHD patients is shown. els and/or activity due to early menopause or as a consequence of antiestrogenic treatment for breast cancer evidenced a rapid and sharp worsening of clinical symptoms during and/or at the end of the treatment (Supplemental Clinical Data, Supplemental Table 2, and Supplemental Figure 5), supporting the existence of a link between estrogen and FSHD. In comparison, relatives carrying the same pathologic D4Z4 contraction as well as patients diagnosed with other types of cancer and undergoing surgery/chemotherapy did not show a similar worsening of clinical parameters (Supplemental Tables 2 and 3 ).

Estrogen antagonizes transcriptional induction of DUX4 target genes. Previous data show that $\mathrm{E}_{2}$ does not affect the protein levels of exogenous DUX4. To understand the molecular mechanism of $\mathrm{E}_{2}$ activity, we analyzed whether $\mathrm{E}_{2}$ interferes with $D U X 4$ transcription. Analysis of endogenous DUX4 in all FSHD myoblasts showed a progressive increase of its mRNA during differentiation, with a peak of expression around the third day and a decline at 7 days (Figure 5, A-D). As a control, DUX4 was undetectable in all control myoblasts (Figure 5E). These data are in agreement with previous data obtained in fetal FSHD myoblasts and confirm a regulated expression of DUX4 during FSHD muscle differentiation (39). The relative increase of DUX4 was independent of sex and not correlated with basal levels of DUX4 mRNA or differentiation properties of FSHD cell cultures (see Figure 1, M and N). Importantly, $\mathrm{E}_{2}$ treatment did not reduce levels of DUX4 (Figure 5F), suggesting that $\mathrm{E}_{2}$ may interfere with DUX4 activity rather than with its expression. Since DUX4 is a transcription factor, we considered the expression levels of TRIM 43 and ZSCAN4, 2 canonical DUX4 target genes $(17,40,41)$. The expression of these genes was highly increased along the differentiation time course (Figure 6, A and B) and approximately paralleled the increase of DUX4, supporting their activation by DUX4 during FSHD myoblast differentiation. Consistently, TRIM43 and ZSCAN4 were not similarly induced in control samples (Figure 6C). It is noteworthy that analysis of these transcripts near their maximum point of expression (5 days) showed a significant reduction by $\mathrm{E}_{2}$ in FSHD myoblasts, but not in control cells (Figure 6, D and E), indicating that $\mathrm{E}_{2}$ is able to interfere with DUX4 transcriptional activity. To further support these data, TRIM43 and ZSCAN4 mRNAs were analyzed in myoblasts overexpressing Cherry-DUX4. In fact, $\mathrm{E}_{2}$ was able to strongly reduce the expression of these genes induced by DUX4, whereas it was ineffective in control cells transfected with mCherry vector (Figure 7, A and B). Overall, these data demonstrate that $\mathrm{E}_{2}$ impairs DUX4 transcriptional activity. To obtain further insight into the molecular mechanism that underlies this phenomenon, we analyzed the occupancy of DUX4 on the promoter of its target, ZSCAN4. Immunoprecipitation of Cherry-DUX4 efficiently and specifically coimmunoprecipitated the DNA at the levels of the ZSCAN4 promoter (Figure $7 \mathrm{C}$ ). Of note, $\mathrm{E}_{2}$ strongly reduced the levels of coimmunoprecipitated ZSCAN4 promoter, suggesting that estrogen indeed antagonizes DUX4 binding to the chromatin, thus reinforcing previous data (Figure $7 \mathrm{C}$ ). As a further indication, trimethylated lysine 4 of histone 3 (H3[Lys4]), a marker of transcriptional activation, showed a striking reduction of chromatin occupancy on the ZSCAN4 promoter in the presence of $\mathrm{E}_{2}$ treatment. Conversely, the levels of an unrelated gene, the protooncogene $M D M 2$, were not affected by $\mathrm{E}_{2}$, indicating the specific activity of the hormone toward DUX4 targets. To confirm 

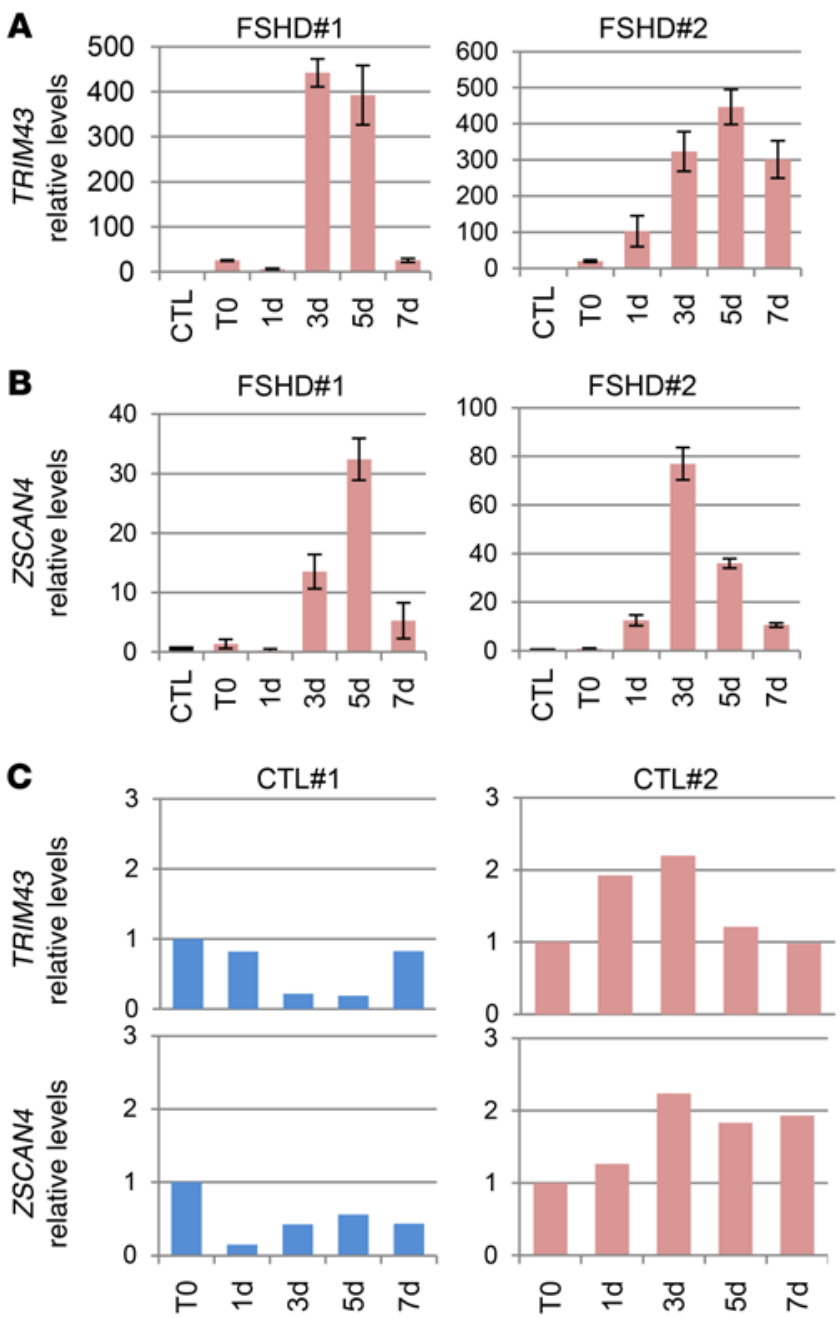
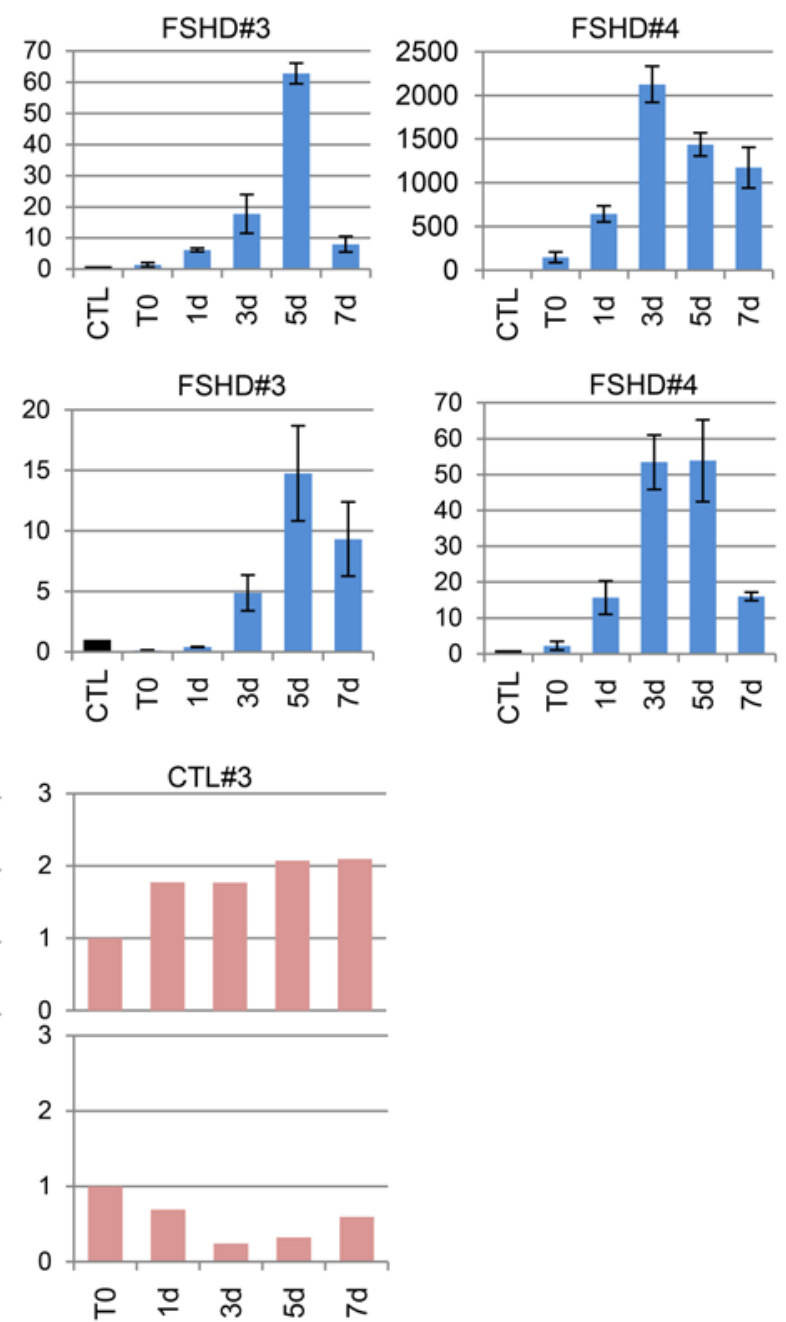

D

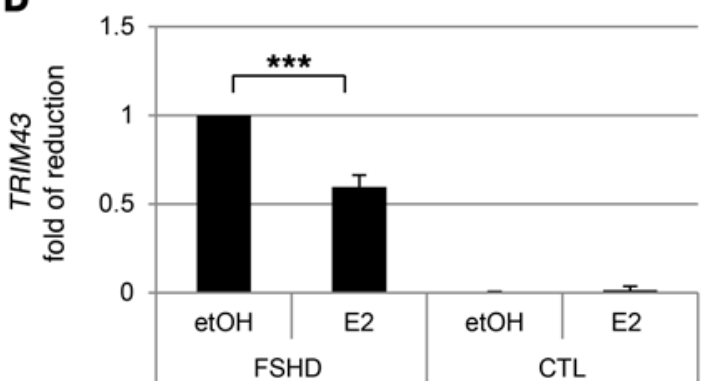

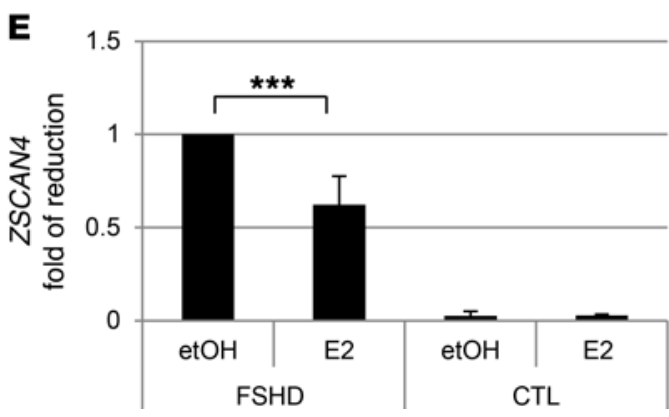

Figure 6. Estrogens antagonize induction of DUX4 targets. (A and B) mRNA levels by qRT-PCR of TRIM43 (A) and ZSCAN4 (B) in immortalized myoblasts from indicated patients, cultured in differentiation medium without estrogens at the indicated time points. The mRNA levels of each gene in proliferating myoblasts from CTL\#1 (time point 0 [TO]) were arbitrarily set to 1 . All samples were normalized to GAPDH. (C) mRNA levels by qRT-PCR of TRIM43 and ZSCAN4 in immortalized myoblasts derived from healthy individuals grown as in $\mathbf{A}$ and $\mathbf{B}$. The mRNA levels at the time point 0 were set to 1 . (A-C) Mean \pm SD of 2 independent experiments is shown. (D and E) Fold reduction of TRIM43 (D) and ZSCAN4 (E) levels in FSHD and CTL myoblasts collected after 5 days of culture in differentiation medium in the absence (etOH) or presence of $\mathrm{E}_{2}$. The mRNA levels upon etOH treatment in FSHD cells were set to 1 . ${ }^{* *} P<0.001$, 1-sample $t$ test.

these data in FSHD myoblasts, endogenous DUX4 or H3(Lys4) was immunoprecipitated using FSHD\#4 cells. In this case too, $\mathrm{E}_{2}$ strongly reduced the levels of the coimmunoprecipitated ZSCAN4 promoter, but did not reduce coimmunoprecipitation of a control gene, MDM2 (Figure 7D), confirming the ability of the hormone to reduce the transcriptional function of endogenous DUX4.
Pathophysiology of FSHD myoblasts has been associated with the expression of PITX1 and of atrogin-1 (also known as FBX032), 2 genes involved in muscle impairment and atrophy and whose transcription has been reported to be increased by DUX4 $(33,42$, 43). To confirm the function of $E_{2}$ in FSHD muscle impairment and DUX4 activity, the expression levels of these 2 genes were 
A

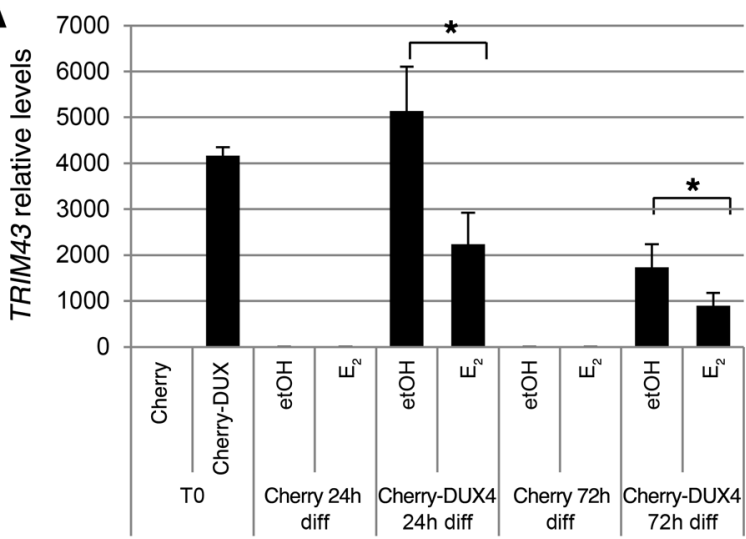

B

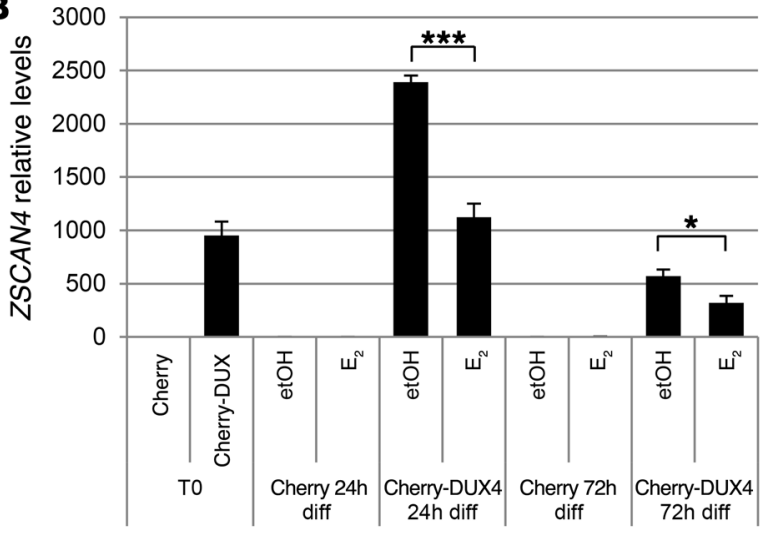

C

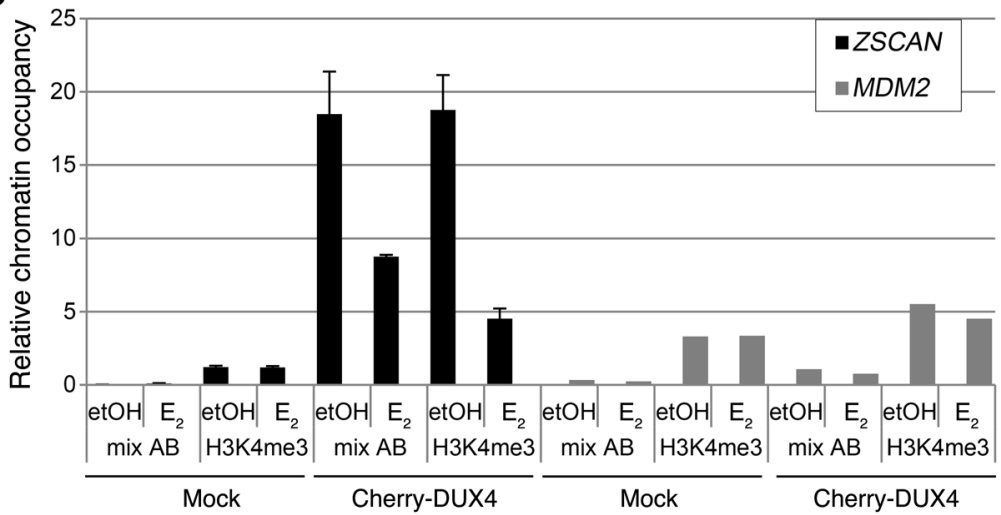

D

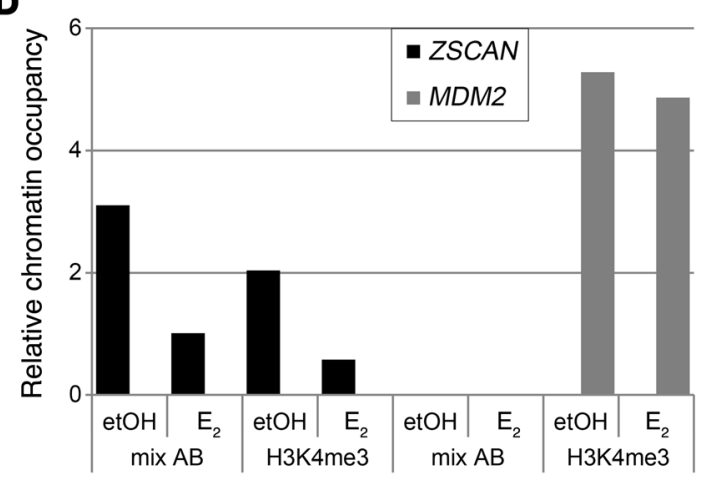

Figure 7. Estrogens antagonize DUX4 transcriptional activity. (A and B) mRNA levels by qRT-PCR of TRIM43 (A) and ZSCAN4 (B) in control myoblasts overexpressing mCherry or Cherry-DUX4 and grown in proliferation medium without estrogen (TO) or in differentiation medium without (etOH) or with $E_{2}$ for the indicated time points. All samples were normalized to GAPDH. Mean \pm SD of 3 independent experiments is shown $(n=3)$. ${ }^{* *} P<0.001 ;{ }^{*} P<0.05,2$-tailed Student's $t$ test. (C) ChIP qPCR analysis of ZSCAN4 and MDM2 promoters following immunoprecipitation of Cherry-DUX4 (with aDUX4/aCherry antibodies $\mathrm{mix}$ ) or H3K4me3 in control cells overexpressing mock or Cherry-DUX4 and cultured in differentiation medium for 24 hours in the absence or presence of $E_{2}$. Mean \pm SD of 2 technical replicates for ZSCAN4 is reported. (D) ChIP qPCR analysis of ZSCAN4 and MDM2 promoters following immunoprecipitation of DUX4 (with aDUX4) or H3K4me3 in immortalized FSHD\#4 myoblasts after 5 days of differentiation. The results are represented as percentage of input.

analyzed as well. Indeed, PITX1 and atrogin-1 showed the same behavior as TRIM43 and ZSCAN4 in FSHD cells (Figure 8, A and B), but not in control myoblasts (Figure $8 \mathrm{C}$ ). Importantly, $\mathrm{E}_{2}$ significantly decreased levels of PITX1 and atrogin-1, but it was ineffective in control cells (Figure 8, D and E).

Overall, these data demonstrate that estrogens antagonize DUX4 transcriptional activity and its differentiation inhibitory function and support the protective role of these hormones toward FSHD myoblast in in vitro differentiation.

$E_{2}$ treatment changes DUX4 intracellular localization. Previous data indicate that $\mathrm{E}_{2}$ reduces DUX4 residency on the promoter of its target. To understand the molecular mechanism, we analyzed DUX4 levels in subcellular fractions. Strikingly, DUX4-V5 levels were strongly decreased by $\mathrm{E}_{2}$ treatment in the chromatin-enriched fraction coincident with its appearance in the cytoplasmic fraction (Figure 9, A and B). Interestingly, this occurred only in the differentiating cells, whereas $\mathrm{E}_{2}$ was ineffective in relocalizing DUX4 in proliferating myoblasts (Figure 9A). Immunofluorescence experiments confirmed that $\mathrm{E}_{2}$ increased cytoplasmic localization of DUX4 upon differentiation, whereas it was ineffective in proliferating myoblasts independently of the DUX4 expression vector used (Figure 9, C and D). Immunofluorescence also confirmed the cytoplasmic localization of DUX4 in proliferating myoblasts and its progressively increased nuclear localization upon differentiation stimulus (Figure 9, C and D). Of importance, analysis of endogenous DUX4 in primary FSHD3 and FSHD4 myoblasts confirmed its nuclear localization after 4 days of growth in differentiation medium lacking estrogens, whereas the signal appeared diffused in the cell or even missing in the nucleus in $\mathrm{E}_{2}$-treated cells (Figure 9E). Overall, these data indicate that estrogens antagonize DUX4 pathogenicity by relocalizing it in the cytoplasm, thereby providing a molecular mechanism for the ability of these hormones to improve FSHD myoblast differentiation.

\section{Discussion}

FSHD is an autosomal dominant neuromuscular disease with extreme variability in the clinical symptoms. One of the factors affecting disease severity is sex, with women being more protected. Our data demonstrate that estrogens improve the differentiation properties of myoblasts isolated from FSHD individuals. These effects are not due to a trophic activity of estrogens on myoblast pro- 
A
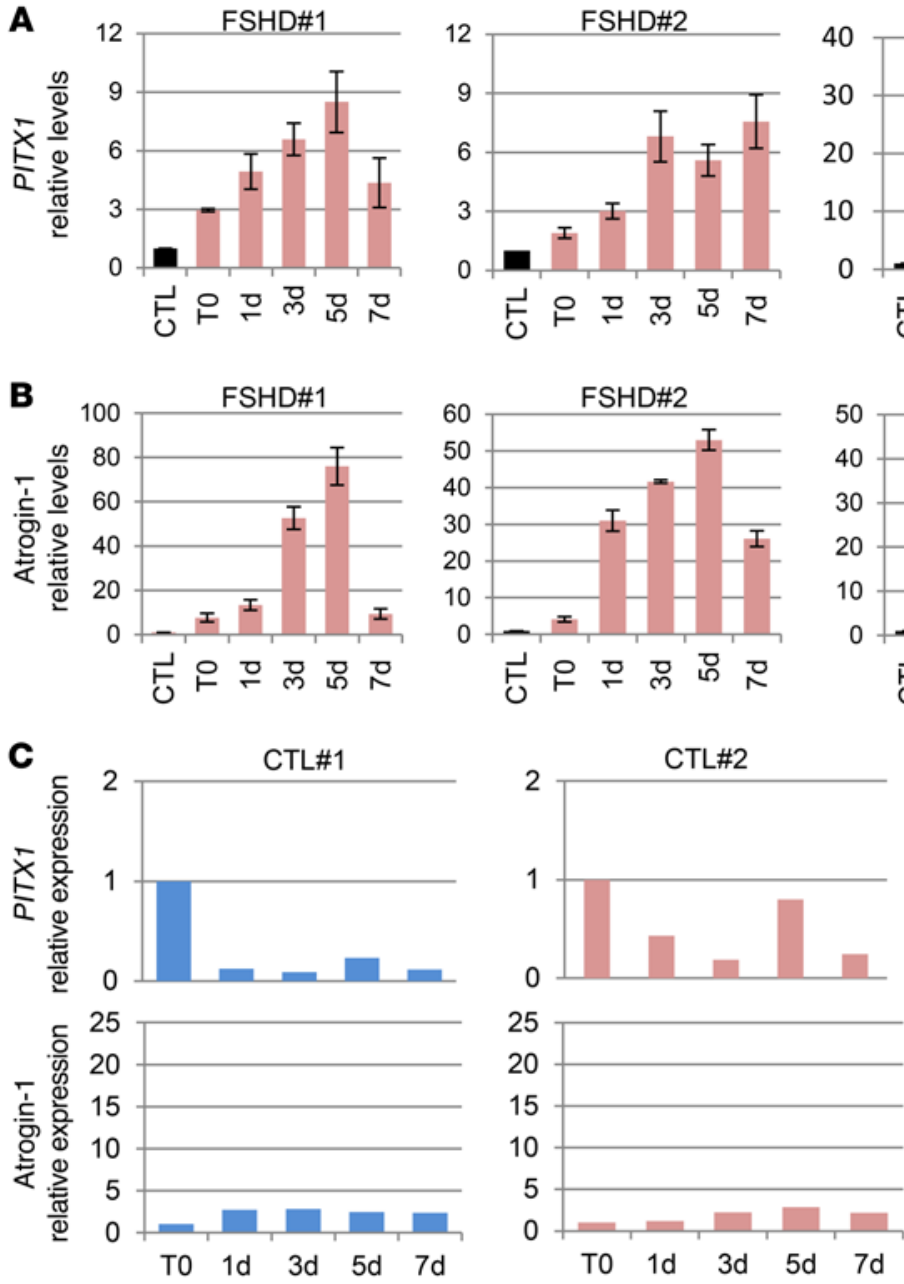

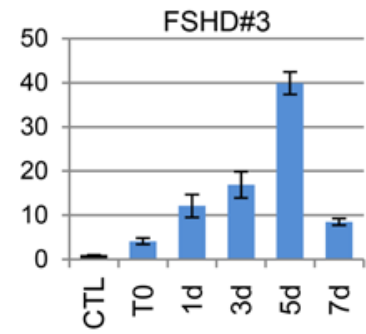

FSHD\#4
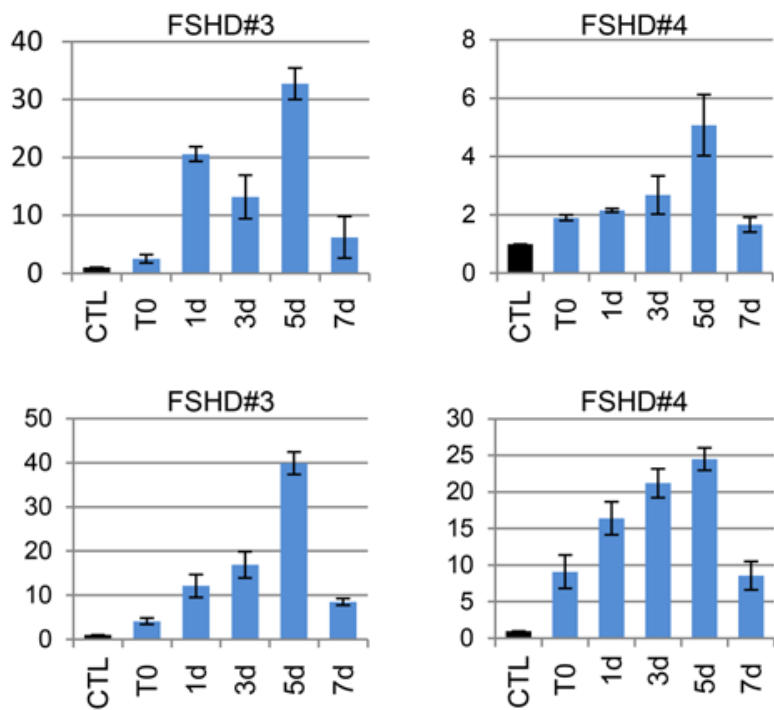
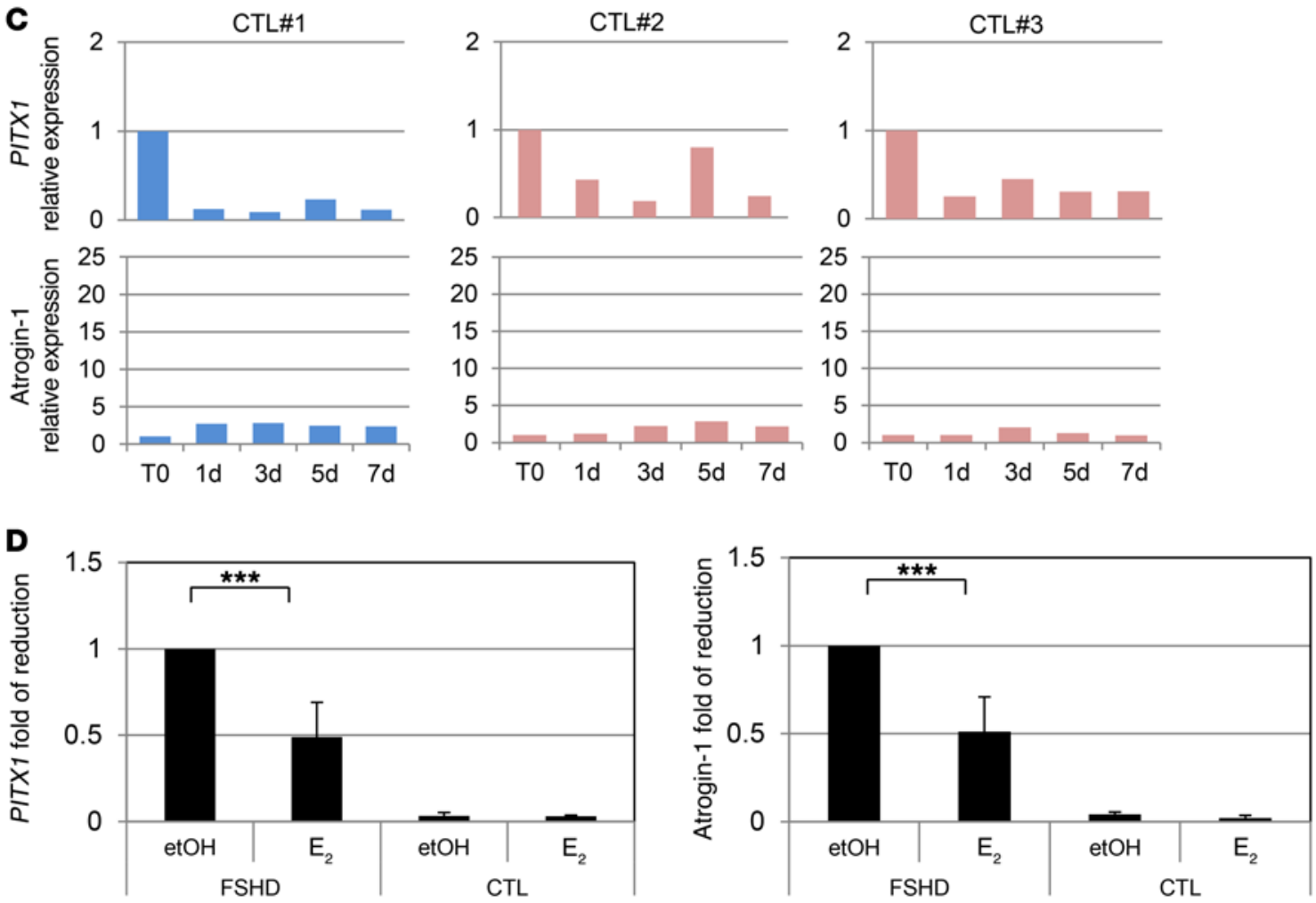

Figure 8. Estrogens antagonize induction of genes involved in FSHD. (A and B) mRNA levels by qRT-PCR of PITX1 (A) and atrogin-1 (B) in immortalized myoblasts derived from indicated patients cultured in differentiation medium without estrogens at the indicated time points. The mRNA levels of each gene in proliferating myoblasts from CTL\#1 (TO) were arbitrarily set to 1. All samples were normalized to GAPDH. (C) mRNA levels by qRT-PCR of PITX1 and atrogin-1 in myoblasts from healthy individuals grown as in $\mathbf{A}$ and $\mathbf{B}$. The mRNA levels at the time point 0 were set to 1 . (A-C) Mean \pm SD of 2 independent experiments is shown. (D and E) Fold of reduction of PITX1 (D) and atrogin-1 (E) levels in FSHD and control myoblasts collected after 5 days of culture in differentiation medium in the absence (etOH) or presence of $\mathrm{E}_{2}$. The mRNA levels upon etOH treatment in FSHD were set to $1 .{ }^{* * *} P<0.001,1-\mathrm{sample} t$ test.

liferation or survival. Conversely, estrogens counteract the downstream effects of misexpressed DUX4, the gene mostly involved in FSHD pathophysiology. Indeed, estrogens antagonize the muscle differentiation impairment caused by DUX4 expression.

The beneficial effects of estrogens on FSHD myoblasts are mediated by ER $\beta$, whereas ER $\alpha$ is not expressed in these cells. Conversely, ER $\alpha$ is expressed in the murine myoblast cell line
$\mathrm{C} 2 \mathrm{C} 12$ and in murine muscles, thus making it necessary to exercise caution when comparing data from murine and human tissues. Of interest, the ER $\beta$ agonist 3 $\beta$-diol is highly effective in antagonizing DUX4-mediated activity. Since this hormone is a metabolite of dihydrotestosterone (DHT) and is, therefore, present in male individuals, its endogenous levels might be an additional factor affecting FSHD clinical severity. 
A

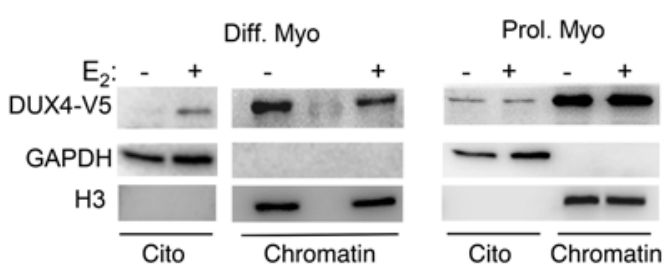

B

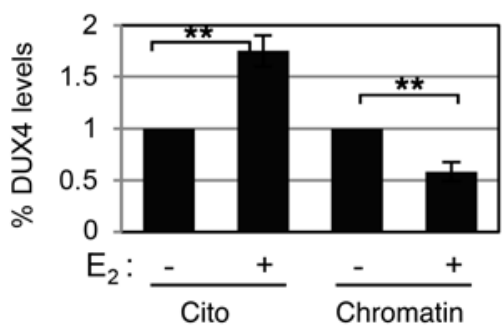

C

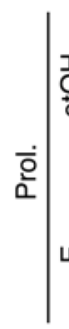

DAPI
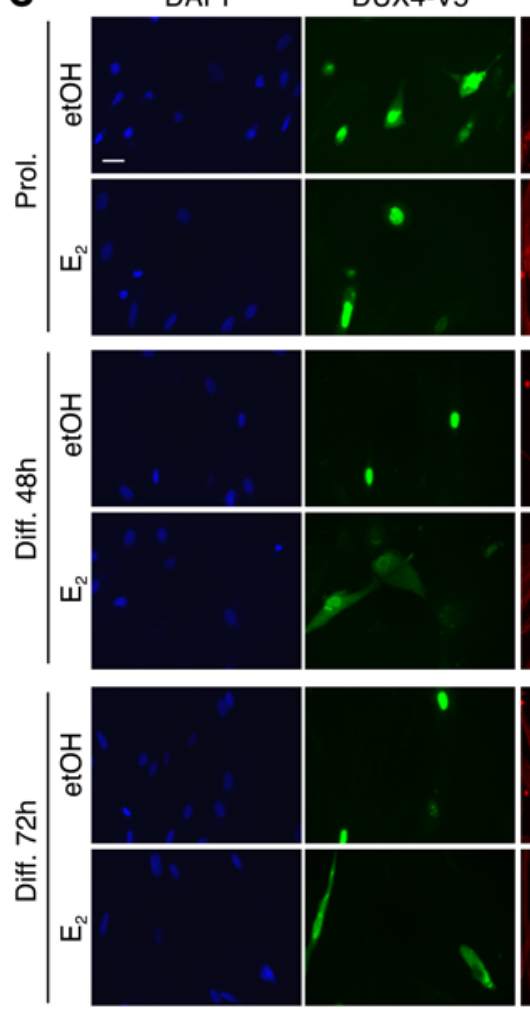

F-actin
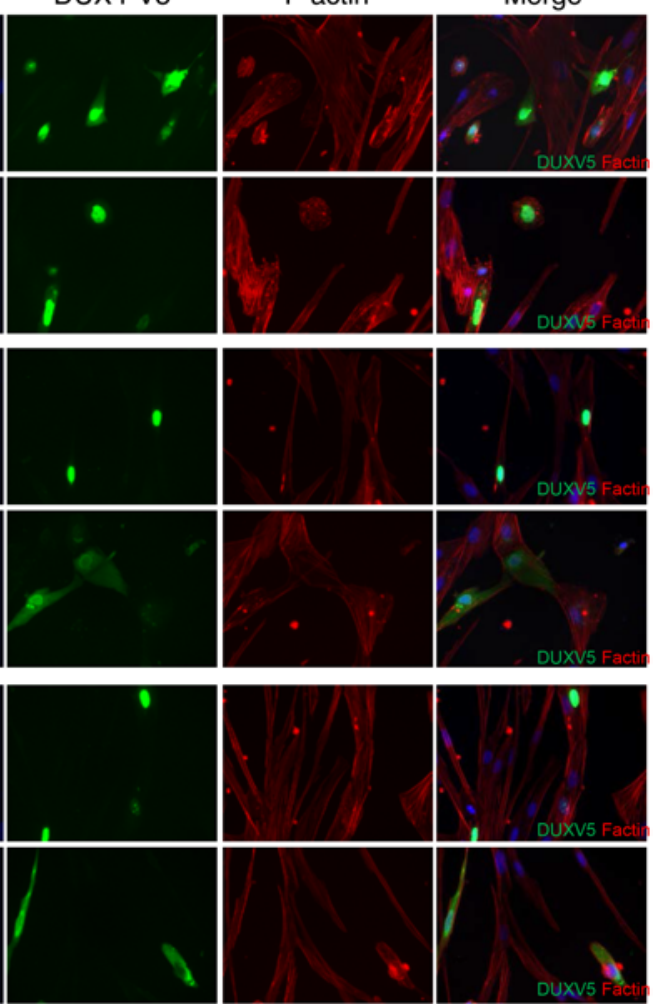

D

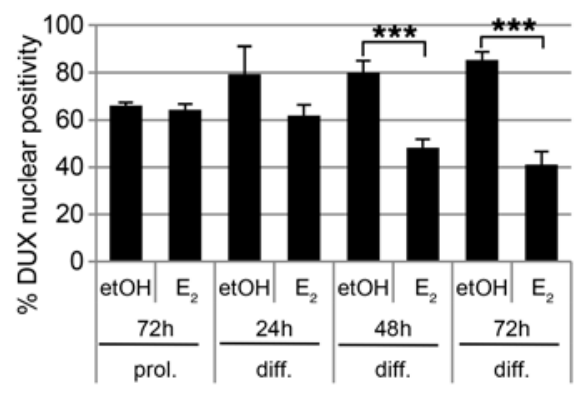

E

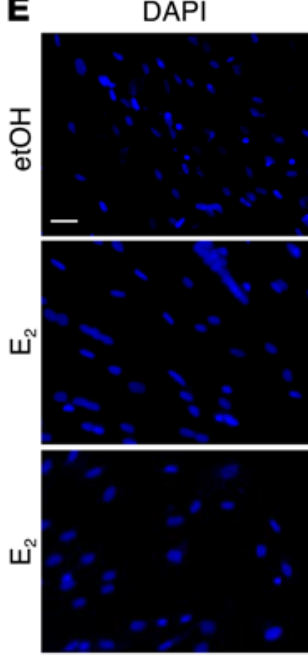

DUX4

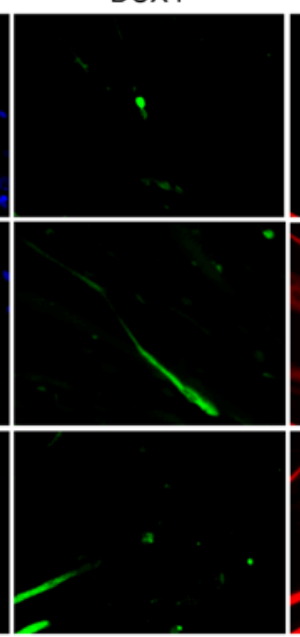

$\mathrm{MHC}$

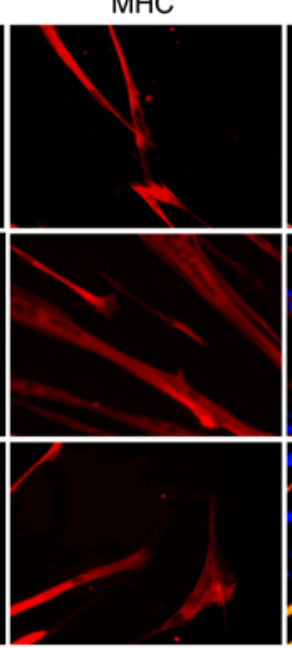

Merge

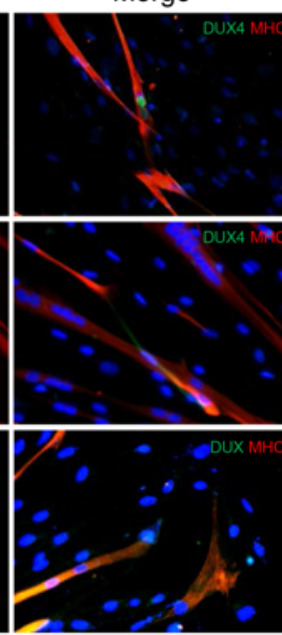

Figure 9. Estrogens change intracellular localization of DUX4. (A) Western blot analysis of indicated proteins in the cytoplasmic (Cito) or chromatin-enriched fraction (chromatin) of immortalized control myoblasts overexpressing DUX4-V5 cultured in differentiation medium for 3 days (Diff. Myo) or in proliferation medium (Prol. Myo) in the absence (etOH) or presence of $E_{2}$. See complete unedited blots in the supplemental material. (B) Quantification of DUX levels analyzed as in $\mathbf{A}$. The signal of DUX4 in the absence of $E_{2}$ was arbitrarily set to 1 . Mean \pm SD of 3 independent experiments is shown ( $n=3$ ). ${ }^{*} P<0.01$, 1-sample $t$ test. (C) Representative photographs of $\mathrm{V} 5$ immunostaining (green) in CTL\#1 myoblasts overexpressing DUX-V5 during proliferation or differentiation at the indicated time points upon etOH or $\mathrm{E}_{2}$ treatment. Cytoplasm is marked by F-actin signal (red). DAPI counterstains nuclei (blue). Scale bar: $25 \mu \mathrm{m}$. (D) Percentages of DUX4 nuclear positive myoblasts treated as in C. Mean \pm SD of 3 independent experiments is shown. Eight different fields/condition were counted $(n=24)$. ${ }^{* *} P<0.001$, 2-tailed Student's $t$ test. (E) Representative photographs of endogenous DUX4 (green) and MHC (red) in primary FSHD\#3 and FSHD\#4 myoblasts cultured in differentiation medium for 4 days in the presence of etOH (FSHD\#4) or $E_{2}$ (middle panels, FSHD\#4; lower panels, FSHD\#3). DAPI counterstains nuclei (blue). Scale bar: $25 \mu \mathrm{m}$.

At the molecular level, our data show that estrogens reduce DUX4 transcriptional function during myoblast differentiation. Of note, we observed that DUX4 levels and activity increase during myotube formation, thus providing a plausible explanation for the increased toxicity of this gene in differentiated compared with proliferating myoblasts. The mRNA levels of TRIM43, ZSCAN4, PITX1, and atrogin-1 increase as well and parallel the increase of DUX4 in differentiating myoblasts of FSHD patients, but not 
of healthy individuals, supporting their role as DUX4 targets in FSHD. Some authors report the lack of correlation between DUX4 and PITX1 levels in FSHD myoblasts, questioning the existence of a direct link between these 2 genes (44). Our observations may reconcile these reports by showing a link during muscle differentiation, but not in proliferating myoblasts. Consistently, Klooster et al. showed increased expression of PITX1 in FSHD myotubes, but not in FSHD myoblasts, compared with controls (44). However, the lower induction of PITX1 compared with TRIM43 and ZSCAN4 as well as its noncanonical DUX4-responsive elements (41) may suggest an indirect link and/or indicate the requirement of additional cofactor or cofactors for its transcription.

Estrogens antagonize DUX4 transcriptional activity at least in part by displacing it from chromatin. The ability of estrogen to relocalize cellular factors has already been reported, although the mechanism by which this ER activity occurs has not been elucidated (45). Data from our group exclude a direct binding between ER $\beta$ and DUX4 independently of cell growth conditions (data not shown), suggesting that ER $\beta$ may modulate cellular factors that in turn affect DUX4 nucleocytoplasmic shuttling during myoblast differentiation. Although nuclear localization signals (NLS) have been recognized in DUX4, only large deletion mutants of the protein alter its intracellular localization (46), suggesting that DUX4 cellular localization is affected by other factors rather than from its specific NLS.

Our results are the first evidence, to our knowledge, of a progressive nuclear localization of DUX4 during the early steps of muscle differentiation. This phenomenon represents an additional factor that contributes to modulating DUX4 activity and related toxicity and suggests the possibility of interfering with DUX4 pathogenicity by other approaches. Accordingly, some authors have shown that cytoplasmic localization of DUX4 is associated with the impairment of its function (46).

The existence of a link between estrogen and the FSHD is supported by the analysis of a limited number of FHD patients (Supplemental Clinical Data). Indeed, this retrospective analysis suggests that a rapid loss of estrogen levels and/or activity in female patients may contribute to worsening clinical symptoms, thus contributing to disease variability, especially in those FSHD patients carrying a fragment size associated with incomplete penetrance of the disease. These conclusions are preliminary, and extensive clinical studies will be required to establish the beneficial effects of estrogens in FSHD patients.

Our data do not exclude that other adult hormones may affect FSHD. Particularly, given the equilibrium existing between female and male steroid hormones, namely estrogens and androgens, it is not excluded that androgens may have a counteractive function, worsening FSHD symptoms in males. However, the common knowledge of the positive activity of the androgen receptor toward the enlargement of skeletal muscle cells and fusion of myoblasts contrasts with the worse clinical symptoms associated with FSHD male patients.

Overall, these results point to estrogens as a potentially important factor affecting FSHD. Since effective pharmacologic treatments that improve patient symptoms and/or slow disease progression have not yet been reported (47), these data may be helpful in approaching therapy-decision strategies as well as in investigating novel hormone-based therapies for FSHD.

\section{Methods}

Cell cultures, constructs, transfections, and treatments. Myoblasts from healthy controls CTL\#1 and CTL\#2 were purchased from Lonza. Myoblasts from healthy controls 03U, 09U, 12U, and from FSHD 03A, 05B, 12A, and 12B were from C. Emerson (University of Massachusetts Medical School, Worcester, Massachusetts, USA). Myoblasts from healthy controls CTL\#3, CTL\#10, and CTL\#14 and from patients FSHD\#1, FSHD\#2, FSHD\#3, FSHD\#4, FSHD\#10, and FSHD\#20 were from the Institute of Neurology, Catholic University. None of the patients received either steroids or immunosuppressive therapy before biopsies. In each patient, the overall clinical severity was assessed using the Clinical Severity Scale (CSS), assigning a score of 2 or less when the disease involved only facial and scapular muscles and a score of 3 (mildly affected), 3.5 (moderately affected), or 4-5 (severely affected) when pelvic and lower limb muscles were involved (4). Cells were isolated using the explantation reexplantation method (48). Briefly, 2- $\mathrm{mm}^{3}$ muscle fragments were placed on the bottom of a Petri dish coated with a $1 \%$ gelatin solution containing human plasma. Cells were maintained in a replicative state for approximately 7 passages using a medium containing $15 \%$ serum and a cocktail of growth factors. During the experiments, all the myoblasts were maintained in SkGM-2 medium (Lonza). For estrogen starvation, cells were incubated overnight in DMEM without phenol red supplemented with glutamine, human EGF ( $25 \mathrm{ng} / \mathrm{ml}), 10 \%$ charcoal/dextran-treated FBS (HyClone), gentamicine, and amphotericine B. The differentiation medium was DMEM/F12 without phenol red supplemented with $1 \%$ charcoal/dextran-treated horse serum. Treatment was with the following: $10 \mathrm{nM} \mathrm{E}_{2}, 100 \mathrm{nM}$ tamoxifen, $100 \mathrm{nM}$ diarylpropionitrile (DPN), and $100 \mathrm{nM} 3 \beta$-diol.

The immortalization procedure consisted of transduction of myoblast cultures with 2 retroviral constructs produced in 293T packaging cells carrying the hTERT gene (provided by S. Bacchetti, McMaster University Hamilton, Ontario, Canada) and the CDK4-R24C variant (Addgene) plus the antibiotic resistance genes puromycin and hygromycin. The transduced cells were selected by culture with puromycin and hygromycin.

Transient transfection was performed using JetPrime reagent (Polyplus) according to the manufacturer's instructions. Cherry-DUX4 was obtained by inserting DUX4 coding sequence in pLV-mCherry plasmid (Addgene) using BrsgI and SalI restriction enzyme sites in frame with mCherry-tag. AAV.CMV.DUX4-V5 construct was obtained by S.Q. Harper (The Ohio State University School of Medicine, Columbus, Ohio, USA). All constructs were checked by sequencing.

Luciferase assays were performed by transient transfection of ERE3-tk-luc plasmid (M. Brown, Dana Farber/Harvard Cancer Center, Boston, Massachusetts, USA) in the presence or absence of pcDNA-FLAG-ER $\beta$ expression plasmid (Addgene) and using the Luciferase Assay Systems (Promega).

Cell proliferation was evaluated by CellTiter-Blue assay (Promega), a homogenous assay that measures the conversion of resazurin to resorufin by metabolically active cells. Briefly, $3 \times 10^{3}$ myoblasts/well were plated in a 96-multiwell plate and after 24 hours grown in starvation medium for 16 hours. Then the cells were cultured in differentiation medium in the absence or presence of $E_{2}$. At different time points, CellTiter-Blue was added to the well, and after 2 hours, absorbance at 570 and $600 \mathrm{~nm}$ were evaluated. 
Immunofluorescence. 300 cells $/ \mathrm{mm}^{2}$ were plated for each cell line. At different time points, cells were fixed in $80 \%$ acetone, blocked in $5 \%$ BSA, then immunodecorated with primary antibodies $\alpha$-desmin (Santa Cruz Biotechnology Inc., catalog sc-58745, clone DE-R-11) and $\alpha$-MHC (clone MF20) (49). For V5 tag staining, cells were fixed in $4 \%$ formaldehyde, permeabilized with $0.05 \%$ Triton X-100, blocked with $5 \%$ BSA, and then incubated with $\alpha-\mathrm{V} 5$ antibody (Santa Cruz Biotechnology Inc.). Cytoplasm was counterstained with phalloidin (tebubio). For endogenous DUX4, cells were fixed with $2 \%$ formaldehyde and permeabilized with $1 \%$ Triton $\mathrm{X}-100$, then incubated with mixture of $\alpha$-DUX4 (Abcam, catalog 124699, clone E5.5) 1:250 and $\alpha$-MHC (clone MF20) overnight at $4^{\circ} \mathrm{C}(50)$. Secondary antibodies used were as follows: Cy3-conjugated AffiniPure donkey anti-mouse IgG (Jackson ImmunoResearch, catalog 715165150) Cy2-conjugated AffiniPure donkey anti-mouse IgG (Jackson ImmunoResearch, catalog 715225150), andCy2-conjugated AffiniPure donkey anti-rabbit IgG (Jackson ImmunoResearch, catalog 711225152). Slides were mounted with ProLong/DAPI (Life Technology) and examined by conventional epifluorescence microscope (Olympus BX51) and confocal system TCS-SP5 (Leica Microsystems). Images were captured by a SPOT RT3 camera and elaborated by IAS software. All experiments were evaluated by at least 3 independent observers, one of which was blinded.

RNA preparation and RT-PCR. RNA extraction was carried out with TRIzol reagent following the manufacturer's instructions (Invitrogen). For quantitative reverse-transcription PCR (qRT-PCR), 500 ng of RNA was reverse transcribed using the Gene Amp kit (Applied Biosystems) and subjected to PCR amplification using SYBR Green PCR Master Mix (Bioline) or specific probe/primers in the case of ESR2 and $\beta$-actin.

The following primers were used: forward hGAPDH 5'-GACAAGCTTCCCGTTCTCAG-3', reverse hGAPDH 5'-GAGTCAACGGATTTGGTCGT-3'; $\beta$-actin preformulated assay (Applied Biosystems 4310881E); forward hPITX1 5'-ACATGAGCATGAGGGAGGAG-3', reverse hPITX1 5'-GTTACGCTCGCGCTTACG-3'; forward hAtrogin-1 5'-CCTTTGTGCCTACAACTGAA-3', reverse hAtrogin-1 5'-CTGCCCTTTGTCTGACAGAAT-3'; forward hTRIM43 5'-ACCCATCACTGGACTGGTGT, reverse hTRIM43 5'-CACATCCTCAAAGAGCCTGA; forward hZSCAN4 5'-TGGAAATCAAGTGGCAAAAAC, reverse hZSCAN4 5'-CTGCATGTGGACGTGGAC; forward DUX4 5'-CCAAGGTACCAGCAGACC-3', reverse DUX4 5'-TCCAGGAGATGTAACTCTAATCCA-3'; probe ESR2 5'-/56-FAM/AAGTGCCGA/ ZEN/CGAGCAGCTG/3IABkFQ/-3'; forward ESR2 55'-GTGGGAATGGTGAAGTGTG-3', reverse ESR2 5'-ACTTCTCTTGGCCTTGCC-3'; and forward ESR1 5'-TCCTACCAGACCCTTCAGTGAAGCT-3', reverse ESR1 5'-CATGTCGAAGATCTCCACCATGCC-3'.

Samples underwent 40 amplification cycles, monitored by an ABI Prism 7900 sequence detector (Applied Biosystems). All amplification reactions were conducted in triplicate and the average of threshold cycles was used to interpolate standard curves and calculate transcript amounts using the software SDS version 2.3 (Applied Biosystems) applying the Pfaffl model (51).

Western blot and isolation of cell fractions. For Western blot, cells were lyzed in RIPA buffer (50 mM Tris-Cl, pH 7.5, $150 \mathrm{mM} \mathrm{NaCl}$, $1 \%$ Nonidet P-40, 0.5\% Na deoxycholate, 0.1\% SDS, 1 mM EDTA). Proteins were resolved by SDS-PAGE and then transferred onto PVDF membranes (Millipore). Membranes were developed using the enhanced chemiluminescence kit (ECL Amersham) by the chemilumi- nescence imaging system Alliance 2.7 (UVitec Cambridge) and quantified by the software Alliance V_1607. Primary antibodies were as follows: $\alpha$-DUX4 (Abcam, catalog 124699, clone E5.5), $\alpha$-V5-tag (Santa Cruz Biotechnology Inc., catalog sc-58052), $\alpha$-FLAG (Sigma-Aldrich, catalog A8592), $\alpha$-ER $\beta$ (Genetex catalog 110607), $\alpha$-ER $\alpha$ (Santa Cruz Biotechnology Inc., catalog sc-543) $\alpha$-tubulin (Sigma-Aldrich, catalog T9026, clone DM-1A), $\alpha-\mathrm{H} 3$ (Abcam, catalog AB1791), $\alpha-$ p21 (Santa Cruz Biotechnology Inc., catalog sc-6246), $\alpha$-MDM2 (clone 2A10/ Ab1)(52), $\alpha$-desmin (Santa Cruz Biotechnology Inc., catalog sc-58745, clone DE-R-11), $\alpha$-actin (Sigma-Aldrich, A4700, clone AC40), and $\alpha$-GAPDH (Millipore, catalog ABS16). IgG-HRP-conjugated secondary antibodies were as follows: $\alpha$-rabbit (Santa Cruz Biotechnology Inc., catalog sc-2054) and $\alpha$-mouse (Bio-Rad, catalog 1721011).

For subcellular fractionation, cells were resuspended in lysis buffer A (10 mM HEPES, pH 7.9, $10 \mathrm{mM} \mathrm{KCl}, 1,5 \mathrm{mM} \mathrm{MgCl}, 0,34 \mathrm{M} \mathrm{sac}$ charose, 10\% glycerol, 0.5 mM EDTA, 0.5 mM EGTA, and 0.1\% Triton $\mathrm{X}-100$ ) added with protease inhibitors (Roche) and 0.5 mM PMSF (Sigma-Aldrich). After centrifugation, the supernatants corresponding to the cytoplasm fraction were further clarified by centrifugation at $15000 \mathrm{~g}$ for 15 minutes. Nuclei pellets were washed once in buffer A and twice in PBS before resuspension in buffer B ( $3 \mathrm{mM}$ EDTA, 0.5 mM EGTA) added with protease inhibitor; these lysates were then incubated on ice for 30 minutes. After centrifugation at $2800 \mathrm{~g}$ for 4 minutes at $4^{\circ} \mathrm{C}$, the pellet obtained represents the chromatin-enriched fraction; this pellet was washed as indicated previously, resuspended in buffer B, and sonicated for 30 seconds at $50 \%$ amplitude.

ChIP. For the ChIP of overexpressed DUX4, $12 \times 10^{6}$ cells were grown in starvation medium for 16 hours and then transfected with Cherry-DUX4 plasmid and after 4 hours cultured in differentiation medium with or without $\mathrm{E}_{2}$. The crosslinking was performed after 24 hours of growth in differentiation medium. For the ChIP of endogenous protein in the FSHD\#4 cell line, we used $5 \times 10^{6}$ cells, and the crosslinking was performed after 5 days of growth in differentiation medium. ChIP was performed according to Consalvi et al. (53). Briefly, chromatin was crosslinked with $1 \%$ formaldehyde (Sigma-Aldrich), and then glycine was added to a final concentration of $0.125 \mathrm{M}$. After 5 minutes, cells were washed, scraped with PBS, and centrifuged. Then pellet samples were resuspended in nuclei lysis buffer (50 mM Tris- $\mathrm{HCl}, \mathrm{pH} 8.1,1 \%$ SDS, $10 \mathrm{mM}$ EDTA) on ice for 30 minutes. Sonication was performed using a Bioruptor UCD-200 Sonicator (Diagenode). Chromatin extracts were immunoprecipitated overnight on a rotating platform at $4^{\circ} \mathrm{C}$ with the following antibodies: mix of $\alpha$-DUX4 (Abcam, catalog 124699, clone E5.5) and $\alpha$-mCherry (Thermo Fischer, catalog M11217) to immunoprecipitate overexpressed DUX4, $\alpha$-DUX4 (Abcam, catalog 124699, clone E5.5) for the endogenous protein, and $\alpha$-trimethyl-histone H3 (Lys4) (Millipore, catalog 07-473). No antibody was used as a negative control. Immunoprecipitated chromatin was conjugated with $G$ Protein Magnetic Beads (Invitrogen). After extensive washing, bound DNA fragments were eluted and analyzed by qPCR of ZSCAN4 (17) and MDM2 (54) promoters, using SYBR Green Master Mix (Bioline).

Statistics. Methods used were 2-tailed Student's $t$ test for comparison of pairs and Kruskal-Wallis with Bonferroni's correction for multiple group comparison. A $P$ value of less than 0.05 was considered significant. The software used was Analyse-it (https://analyse-it.com/).

Study approval. All muscle biopsies were performed after informed consent of individuals. The clinical data derive from the routine follow-up of patients treated at the Centre Hospitalier, University of la Cote d'Azur, 
Nice, France. The study does not report on the use of experimental or new protocols and does not involve direct participation of human beings. Given the retrospective nature of the study, there is not a requirement of approval by an institutional review board. Patients and/or their relatives provided the consent for the utilization of the clinical data for this study.

\section{Author contributions}

ET performed most of the experiments. MP performed all the FACS analyzes. SS, A Puma, and MG followed and analyzed the clinical family data. SFV performed the confocal experiments. RM, GT, and ER prepared and characterized the human FSHD myoblasts from Rome. GG and CPT provided and analyzed some of the clinical data. PC, GD, and IC developed and tested the Cherry-DUX4 construct. A Pontecorvi provided a critical evaluation of hormone therapies. ET, MC, GD, and FM discussed and planned the experiments. FM and GD supervised the project. FM wrote the manuscript.

\section{Acknowledgments}

This work was supported by Project "FaReBio di Qualita" from the Italian Ministry of Economy and Finance/CNR (to FM) and by a FSH Society grant (FSHS-82014-01 to GD). ET was supported by a short-term mobility fellowship from the Italian Society of Biophysics and Molecular Biology (SIBBM). We acknowledge A. Farsetti for helpful discussion about ER $\beta$, D. Palacios for support for ChIP experiments, M. Sandri for advice about atrogin-1, and S. Van Der Maarel's group for helpful discussion. During the preparation of this manuscript, Isabella Cascino passed away. We are grief-stricken by her death, and we dedicate this work to her memory.

Address correspondence to: Fabiola Moretti, Institute of Cell Biology and Neurobiology, National Research Council of Italy (CNR), Via del Fosso di Fiorano, 64, 00143 Rome, Italy. Phone: 39.06501703242; E-mail: fabiola.moretti@cnr.it.
1. Tawil R, Van Der Maarel SM. Facioscapulohumeral muscular dystrophy. Muscle Nerve. 2006;34(1):1-15.

2. Statland JM, Donlin-Smith CM, Tapscott SJ, Lemmers RJ, van der Maarel SM, Tawil R. Milder phenotype in facioscapulohumeral dystrophy with 7-10 residual D4Z4 repeats. Neurology. 2015;85(24):2147-2150.

3. Zatz M, Marie SK, Cerqueira A, Vainzof M, Pavanello RC, Passos-Bueno MR. The facioscapulohumeral muscular dystrophy (FSHD1) gene affects males more severely and more frequently than females. Am JMed Genet. 1998;77(2):155-161.

4. Ricci E, et al. Progress in the molecular diagnosis of facioscapulohumeral muscular dystrophy and correlation between the number of KpnI repeats at the $4 \mathrm{q} 35$ locus and clinical phenotype. Ann Neurol.1999;45(6):751-757.

5. Tonini MM, et al. Homozygosity for autosomal dominant facioscapulohumeral muscular dystrophy (FSHD) does not result in a more severe phenotype. JMed Genet. 2004;41(2):e17.

6. Lin F, Wang ZQ, Lin MT, Murong SX, Wang N. New insights into genotype-phenotype correlations in Chinese facioscapulohumeral muscular dystrophy: a retrospective analysis of 178 patients. Chin Med J. 2015;128(13):1707-1713.

7. Klinge L, Eagle M, Haggerty ID, Roberts CE, Straub V, Bushby KM. Severe phenotype in infantile facioscapulohumeral muscular dystrophy. Neuromuscul Disord. 2006;16(9-10):553-558.

8. Lunt PW, et al. Correlation between fragment size at D4F104S1 and age at onset or at wheelchair use, with a possible generational effect, accounts for much phenotypic variation in 4q35-facioscapulohumeral muscular dystrophy (FSHD). Hum Mol Genet. 1995;4(5):951-958.

9. Rudnik-Schöneborn S, Glauner B, Röhrig D, Zerres K. Obstetric aspects in women with facioscapulohumeral muscular dystrophy, limb-girdle muscular dystrophy, and congenital myopathies. Arch Neurol. 1997;54(7):888-894.

10. Awater C, Zerres K, Rudnik-Schöneborn S. Pregnancy course and outcome in women with hereditary neuromuscular disorders: comparison of obstetric risks in 178 patients. Eur J Obstet Gynecol Reprod Biol. 2012;162(2):153-159.
11. Ciafaloni E, et al. Pregnancy and birth outcomes in women with facioscapulohumeral muscular dystrophy. Neurology. 2006;67(10):1887-1889.

12. Sacconi S, Salviati L, Desnuelle C. Facioscapulohumeral muscular dystrophy. Biochim Biophys Acta. 2015;1852(4):607-614.

13. Tawil R, van der Maarel SM, Tapscott SJ. Facioscapulohumeral dystrophy: the path to consensus on pathophysiology. Skelet Muscle. 2014;4:12.

14. Calandra P, et al. Allele-specific DNA hypomethylation characterises FSHD1 and FSHD2. JMed Genet. 2016;53(5):348-355.

15. van Overveld PG, et al. Hypomethylation of D4Z4 in 4q-linked and non-4q-linked facioscapulohumeral muscular dystrophy. Nat Genet. 2003;35(4):315-317.

16. Lemmers RJ, et al. Inter-individual differences in CpG methylation at D4Z4 correlate with clinical variability in FSHD1 and FSHD2. Hum Mol Genet. 2015;24(3):659-669.

17. Geng LN, et al. DUX4 activates germline genes, retroelements, and immune mediators: implications for facioscapulohumeral dystrophy. Dev Cell. 2012;22(1):38-51.

18. Daxinger L, Tapscott SJ, van der Maarel SM. Genetic and epigenetic contributors to FSHD. Curr Opin Genet Dev. 2015;33:56-61.

19. Dieli-Conwright CM, Spektor TM, Rice JC, Todd Schroeder E. Oestradiol and SERM treatments influence oestrogen receptor coregulator gene expression in human skeletal muscle cells. Acta Physiol (Oxf). 2009;197(3):187-196.

20. Wiik A, et al. Expression of oestrogen receptor $\alpha$ and $\beta$ is higher in skeletal muscle of highly endurance-trained than of moderately active men. Acta Physiol Scand. 2005;184(2):105-112.

21. Wiik A, Ekman M, Johansson O, Jansson E, Esbjörnsson M. Expression of both oestrogen receptor $\alpha$ and $\beta$ in human skeletal muscle tissue. Histochem Cell Biol. 2009;131(2):181-189.

22. Wiik A, et al. Oestrogen receptor $\beta$ is expressed in adult human skeletal muscle both at the mRNA and protein level. Acta Physiol Scand. 2003;179(4):381-387.

23. Shiomi K, et al. CDK4 and cyclin D1 allow human myogenic cells to recapture growth property without compromising differentiation potential.
Gene Ther. 2011;18(9):857-866.

24. Barro M, Carnac G, Flavier S, Mercier J, Vassetzky Y, Laoudj-Chenivesse D. Myoblasts from affected and non-affected FSHD muscles exhibit morphological differentiation defects. J Cell Mol Med. 2010;14(1-2):275-289.

25. Tassin A, et al. DUX4 expression in FSHD muscle cells: how could such a rare protein cause a myopathy? J Cell Mol Med. 2013;17(1):76-89.

26. Dmitriev P, et al. DUX4-induced constitutive DNA damage and oxidative stress contribute to aberrant differentiation of myoblasts from FSHD patients. Free Radic Biol Med. 2016;99:244-258.

27. Knopp P, et al. DUX4 induces a transcriptome more characteristic of a less-differentiated cell state and inhibits myogenesis. J Cell Sci. 2016;129(20):3816-3831.

28. Yakimchuk K, Jondal M, Okret S. Estrogen receptor $\alpha$ and $\beta$ in the normal immune system and in lymphoid malignancies. Mol Cell Endocrinol. 2013;375(1-2):121-129.

29. Thomas C, Gustafsson JÅ. The different roles of ER subtypes in cancer biology and therapy. Nat Rev Cancer. 2011;11(8):597-608

30. Lemmers RJ, et al. A unifying genetic model for facioscapulohumeral muscular dystrophy. Science. 2010;329(5999):1650-1653.

31. Wallace LM, et al. DUX4, a candidate gene for facioscapulohumeral muscular dystrophy, causes p53-dependent myopathy in vivo. Ann Neurol. 2011;69(3):540-552.

32. Bosnakovski D, et al. An isogenetic myoblast expression screen identifies DUX4-mediated FSHD-associated molecular pathologies. ЕМВO J. 2008;27(20):2766-2779.

33. Vanderplanck C, et al. The FSHD atrophic myotube phenotype is caused by DUX4 expression. PLoS One. 2011;6(10):e26820.

34. Bosnakovski D, Daughters RS, Xu Z, Slack JM, Kyba M. Biphasic myopathic phenotype of mouse DUX, an ORF within conserved FSHD-related repeats. PLoS One. 2009;4(9):e7003.

35. Sappok A, Mahlknecht U. Ribavirin restores ESR1 gene expression and tamoxifen sensitivity in ESR1 negative breast cancer cell lines. Clin Epigenetics. 2011;3:8.

36. O'Donnell AJ, Macleod KG, Burns DJ, Smyth JF, 
Langdon SP. Estrogen receptor-alpha mediates gene expression changes and growth response in ovarian cancer cells exposed to estrogen. Endocr Relat Cancer. 2005;12(4):851-866.

37. Wijayaratne AL, McDonnell DP. The human estrogen receptor-alpha is a ubiquitinated protein whose stability is affected differentially by agonists, antagonists, and selective estrogen receptor modulators. J Biol Chem. 2001;276(38):35684-35692.

38. Meyers MJ, Sun J, Carlson KE, Marriner GA, Katzenellenbogen BS, Katzenellenbogen JA. Estrogen receptor-beta potency-selective ligands: structure-activity relationship studies of diarylpropionitriles and their acetylene and polar analogues. J Med Chem. 2001;44(24):4230-4251.

39. Ferreboeuf M, et al. DUX4 and DUX4 downstream target genes are expressed in fetal FSHD muscles. Hum Mol Genet. 2014;23(1):171-181.

40. Rickard AM, Petek LM, Miller DG. Endogenous DUX4 expression in FSHD myotubes is sufficient to cause cell death and disrupts RNA splicing and cell migration pathways. Hum Mol Genet. 2015;24(20):5901-5914.

41. Zhang Y, et al. DNA-binding sequence specificity of DUX4. Skelet Muscle. 2016;6:8.

42. Dixit M, et al. DUX4, a candidate gene of facio- scapulohumeral muscular dystrophy, encodes a transcriptional activator of PITX1. Proc Natl Acad Sci U S A. 2007;104(46):18157-18162.

43. Pandey SN, et al. Conditional over-expression of PITX1 causes skeletal muscle dystrophy in mice. Biol Open. 2012;1(7):629-639.

44. Klooster R, et al. Comprehensive expression analysis of FSHD candidate genes at the mRNA and protein level. Eur J Hum Genet. 2009;17(12):1615-1624.

45. Pinto G, et al. Systematic nucleo-cytoplasmic trafficking of proteins following exposure of MCF7 breast cancer cells to estradiol. J Proteome Res. 2014;13(2):1112-1127.

46. Corona ED, Jacquelin D, Gatica L, Rosa AL. Multiple protein domains contribute to nuclear import and cell toxicity of DUX4, a candidate pathogenic protein for facioscapulohumeral muscular dystrophy. PLoS One. 2013;8(10):e75614.

47. Tawil R, et al. Evidence-based guideline summary: Evaluation, diagnosis, and management of facioscapulohumeral muscular dystrophy: Report of the Guideline Development, Dissemination, and Implementation Subcommittee of the American Academy of Neurology and the Practice Issues Review Panel of the American Association of Neuromuscular \& Electrodiagnostic Medicine.
Neurology. 2015;85(4):357-364.

48. Broccolini A, et al. Neprilysin participates in skeletal muscle regeneration and is accumulated in abnormal muscle fibres of inclusion body myositis. J Neurochem. 2006;96(3):777-789.

49. Bader D, Masaki T, Fischman DA. Immunochemical analysis of myosin heavy chain during avian myogenesis in vivo and in vitro. J Cell Biol. 1982;95(3):763-770.

50. Balog J, et al. Increased DUX4 expression during muscle differentiation correlates with decreased SMCHD1 protein levels at D4Z4. Epigenetics. 2015;10(12):1133-1142.

51. Pfaffl MW. A new mathematical model for relative quantification in real-time RT-PCR. Nucleic Acids Res. 2001;29(9):e45.

52. Chen J, Marechal V, Levine AJ. Mapping of the p53 and mdm-2 interaction domains. Mol Cell Biol.1993;13(7):4107-4114.

53. Consalvi S, Brancaccio A, Dall'Agnese A, Puri PL, Palacios D. Praja1 E3 ubiquitin ligase promotes skeletal myogenesis through degradation of EZH2 upon p38 $\alpha$ activation. Nat Commun. 2017;8:13956.

54. Pellegrino M, et al. Targeting the MDM2/MDM4 interaction interface as a promising approach for 553 reactivation therapy. Cancer Res. 2015;75(21):4560-4572. 